\title{
Calibration of thin-film platinum sensors for use in the JILA Sr II Clock
}

\author{
Weston L. Tew \\ Travis L. Nicholson \\ Ross B. Huston
}

This publication is available free of charge from:

http://dx.doi.org/10.6028/NIST.IR.8046

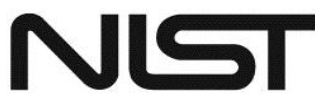


NISTIR 8046

\title{
Calibration of thin-film platinum sensors for use in the JILA Sr II Clock
}

\author{
Weston L. Tew \\ Sensor Science Division \\ Physical Measurement Laboratory \\ Travis L. Nicholoson \\ Ross. B. Huston \\ JILA, University of Colorado \\ Boulder, $\mathrm{CO}$
}

This publication is available free of charge from:

http://dx.doi.org/10.6028/NIST.IR.8046

March 2015

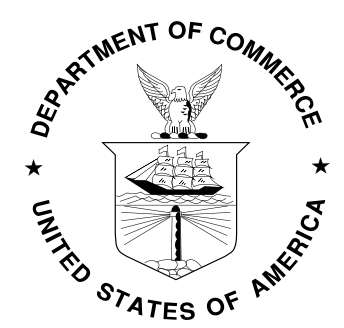

U.S. Department of Commerce Penny Pritzker, Secretary

National Institute of Standards and Technology Willie May, Acting Under Secretary of Commerce for Standards and Technology and Acting Director 


\section{Table of Contents}

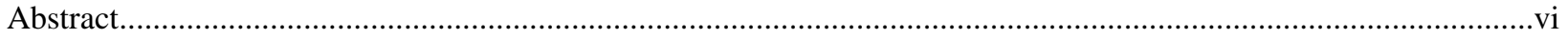

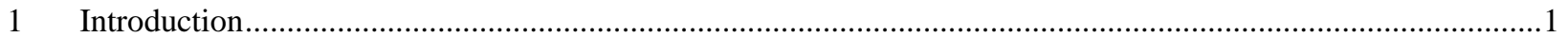

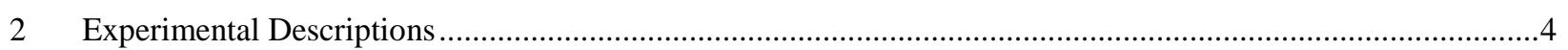

2.1 The NIST Gaithersburg Temperature Calibration Facility.................................................................

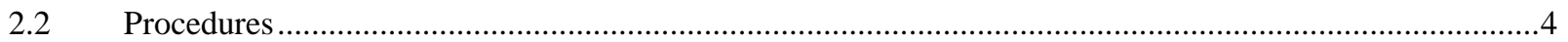

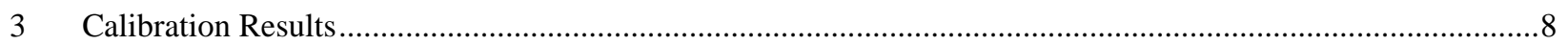

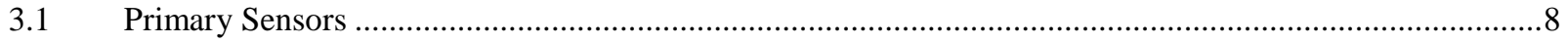

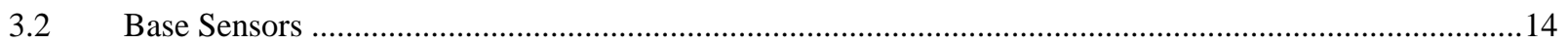

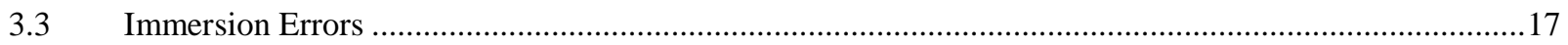

3.4 Use of the Base Sensors for Correcting Immersion Errors .................................................................19

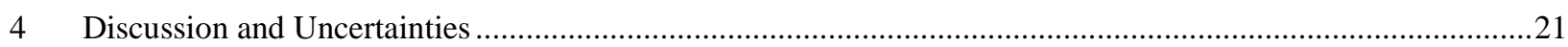

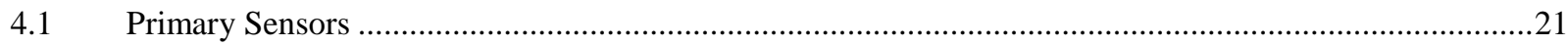

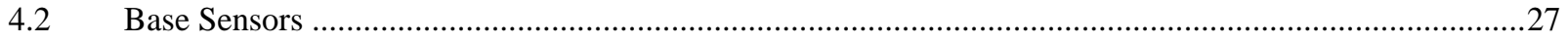

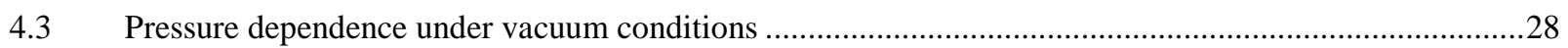

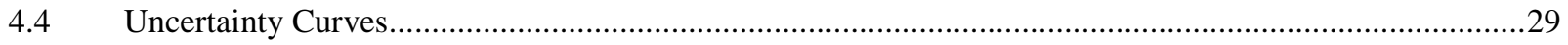

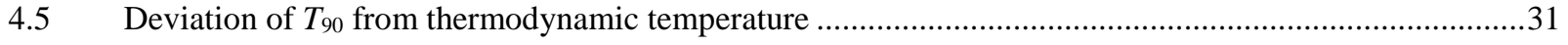

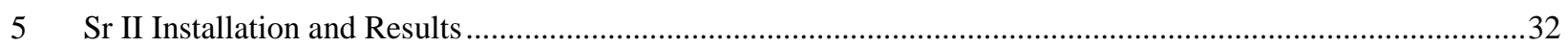

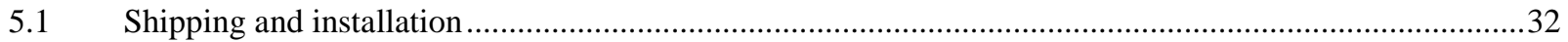

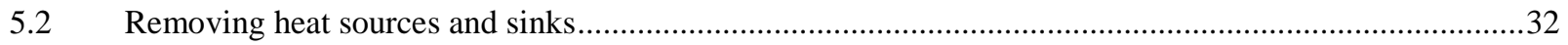

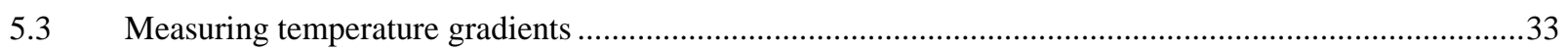

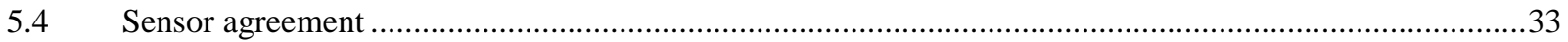

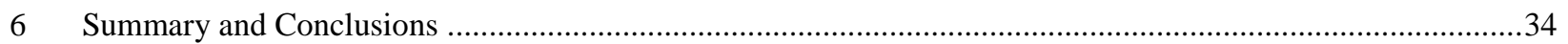

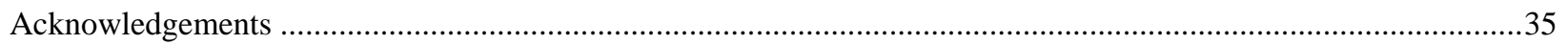

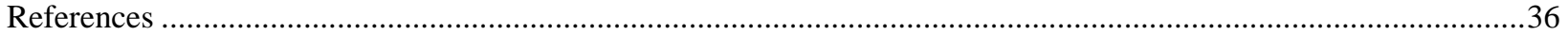




\section{List of Tables}

Table 1. Probe and sensor identifying information.

Table 2. Summary of zero-power resistance data for the primary sensors \# 13 and \# 14. All resistances are in Ohms, all temperatures are in ${ }^{\circ} \mathrm{C}$.

Table 3. Summary of $1 \mathrm{~mA}$ resistance and temperature data for the base sensors, \#15 and \#16. All resistances are in Ohms, and temperatures in ${ }^{\circ} \mathrm{C}$.

Table 4. Summary of the self-heating coefficients $\eta$ for the two primary sensors \#13 and \#14. The units for $\eta$ are $\mathrm{mK} / \mu \mathrm{W}$ and ${ }^{\circ} \mathrm{C}$ for temperature.

Table 5a. Helium calibration parameters for the primary sensors according to Equation 1 using zeropower resistances and fitting all three parameters.

Table 5b. Helium calibration parameters for the primary sensors using $W(t)-1$ and zero-power resistances fitting $A \& B$ parameters only.

Table 6a. $1 \mathrm{~mA}$ Calibration coefficients for the two TF base sensors.

Table 6b. 0 mA Calibration coefficients for the two TF base sensors.

Table 7. The $0 \mathrm{~mA}$ Calibration coefficients for the two WW base sensors.

Table 8. Least-squares fitting estimates for the linear immersion error coefficients for the two probes. The $C_{m n}$ are the immersion error coefficients and $\Delta_{n}$ are ad hoc offset parameters.

Table 9. Recommended values and standard uncertainties for the linear immersion error coefficients and offset parameters for the two probes.

Table 10. Summary of non-parametric uncertainties for the primary and base sensors. 


\section{List of Figures}

Figure 1. The JILA sensor test chamber. a) showing the water-tight shroud covering the probe 1 base and b) showing the chamber in cross section along the mid-plane including the sensor probes. The approximate location of the bath water line is shown as the horizontal blue line ' $\mathrm{W}$ '.

Figure 2. The setup showing the test chamber immersed in the water comparison bath with the SPRTs.

Figure 3. Self-heating plots for both primary sensors at $25^{\circ} \mathrm{C}$ under 2 Torr $(270 \mathrm{~Pa}$ ) He, 7 Torr (930 Pa) Ar, and vacuum $\left(<1 \times 10^{-7}\right.$ Torr $\left.\left(1.3 \times 10^{-5} \mathrm{~Pa}\right)\right)$ from Series 2 .

Figure 4. Deviations of the primary and base sensor resistance calibration data and curve fits from the nominal IEC curve for a Pt100 thermometer. IEC class AA tolerance bands are shown for comparison.

Figure 5. Fit residuals to Eqn. 1 for the two primary sensors. The error bars represent standard statistical (i.e. nominally random) uncertainty only.

Figure 6a. Fit residuals to Eqn. 1 for the two TF base sensors $m=15,16$ for both $1 \mathrm{~mA}$ and $0 \mathrm{~mA}$ data. The error bars represent standard statistical (i.e. nominally random) uncertainty only. The $27{ }^{\circ} \mathrm{C}$ and $40{ }^{\circ} \mathrm{C}$ data were excluded from the $1 \mathrm{~mA}$ fit.

Figure 6b. Fit residuals to Eqn. 1 for the two WW base sensors, $m=17,18$ for $0 \mathrm{~mA}$ data. The error bars represent standard statistical (i.e. nominally random) uncertainty only.

Figure 7. The complete set of immersion errors in Ohms versus the axial gradients $t_{m}-t_{\text {bath }}$ for both He and Ar data.

Figure 8. Linear portions of the Immersion Error curves for the vacuum and helium data.

Figure 9. Primary sensor transient response to step changes in the self-heating power under helium exchange gas. Each point is $90 \mathrm{~s}=0.001 \mathrm{~d}$. Fluctuations are due to bath temperature instability of $\pm 1 \mathrm{mK}$.

Figure 10. Primary sensor transient response to step changes in the self-heating power under vacuum. The increase of $0.5 \mathrm{~m} \Omega(1.3 \mathrm{mK})$ on the return to the base power level of $4 \mu \mathrm{W}$ is due to drift in room temperature during the elapsed $1.9 \mathrm{~h}$.

Figure 11. Self-heating coefficients of the primary sensors in helium, argon and vacuum.

Figure 12. Axial coordinates for the probe immersions. Note the coordinate $x$ is negative for upward displacements. The difference in the effective immersion lengths $L$ for the two probes is exaggerated, but deliberate (see text).

Figure 13. Relationship for the system pressure and internal test chamber pressure.

Figure 14. The equilibration of the primary sensors during a pump out from helium to vacuum.

Figure 15a. Standard uncertainty components and combined total uncertainties in measured temperature $t_{13}$ when using probe 1 with the $n=13$ sensor to determine temperature in the Sr II vacuum chamber.

Figure 15b. Standard uncertainty components and combined total uncertainties in measured temperature $t_{14}$ when using probe 2 with the $n=14$ sensor to determine temperature in the Sr II vacuum chamber. 
Figure 16. The sensors inside the Sr II chamber. Here heat sources and sinks have been removed and passive temperature stabilization is employed to make the temperature uniform inside the chamber. The standard deviation of the data (shown as a gray confidence band in the plot) indicates that the ambient temperature is stable to $\pm 130 \mathrm{mK}$

Figure 17. The difference between the long and short sensors. The measured difference between the sensors is within their combined uncertainty of $\pm 12 \mathrm{mK}$. 


\title{
Calibration of thin-film platinum sensors for use in the JILA Sr II Clock
}

\author{
W. L. Tew ${ }^{1}$, T. L. Nicholson ${ }^{2}$ and R. B. Hutson ${ }^{2}$ \\ ${ }^{1}$ Sensor Science Division, NIST, Gaithersburg, MD \\ ${ }^{2}$ JILA, U. Colorado, Boulder, CO
}

$\begin{array}{ll}\text { Prepared For: } & \text { Dr. Jun Ye, Fellow } \\ & \text { JILA / NIST Quantum Physics Division } \\ & \text { University of Colorado, Boulder, CO }\end{array}$

\begin{abstract}
Thin -film platinum resistance thermometers (TFPRTs) have been calibrated on the International Temperature Scale of 1990 (ITS-90) for the purposes of determining temperatures inside the JILA Sr II Optical Lattice Clock vacuum chamber. The calibration concerns the use of a special test chamber for two high-vacuum immersion probes with two TFPRTs each. The data are presented as a function of temperature, self-heating power, exchange gas / vacuum pressure, and axial gradients. We report the procedures, results, and analysis for these calibrations and estimate the resulting uncertainties for transferring the calibrations and correction equations to the Sr II chamber.
\end{abstract}

Keywords: Temperature calibration; thin-film platinum resistance thermometers; ultra-high vacuum; ITS-90; thermometry; optical lattice atomic clocks; black body radiatio 


\section{Introduction}

The Joint Institute for Laboratory Astrophysics (JILA) has a requirement for determining the temperature under ultra-high vacuum (UHV) conditions as part of the efforts there to improve optical lattice clocks based on arrays of Sr atoms. The Jun Ye group at JILA has developed a new optical lattice clock, the Sr II [1], for this purpose which utilizes a set of Si diode-based temperature probes. The purpose of the probes is to determine the ambient electromagnetic field, due to the blackbody radiation (BBR) emitted by the UHV chamber, that interacts with the $\mathrm{Sr}$ atoms. The BBR interaction creates a systematic bias in the atomic transition frequency upon which the clock is based, an effect that limits many of the best atomic clocks. Determining the BBR field presents certain challenges for any thermal radiation detector due to the relatively weak coupling that exists via BBR at only $300 \mathrm{~K}$. While Si diodes offer certain advantages in radiance responsivity, other measurement systematics concerning reproducibility, self-heating and traceability are less favorable. This has led to an iteration of the probe design where thin film platinum resistance thermometers (TFPRTs) replace the Si diodes. This report provides a detailed account of how these new probes have been calibrated for use in the JILA Sr II optical lattice clock.

Two new sensor probes have been constructed by JILA for use in the Sr II vacuum chamber. The two probes are constructed from borosilicate glass tubing with a $7 \mathrm{~mm}$ diameter and a $1 \mathrm{~mm}$ wall thickness. Both ends of the tubing are sealed for use under UHV conditions in the Sr II vacuum chamber. One end (the 'base') of the glass tubing is sealed against a $6.35 \mathrm{~mm}$ OD stainless steel (SS) tube extension which is welded into a $34 \mathrm{~mm}$ diameter 'Conflat' flange (133CF). The other end of the glass tubing is sealed and terminated with a small $2 \mathrm{~mm}$ diameter glass rod which serves as a mounting surface for a TFPRT. The two sensor probes are designed with different overall lengths. Probe \#1 is approximately $81 \mathrm{~mm}$ in length from the sensor mount point to the interior side of the $133 \mathrm{CF}$ while Probe \#2 is approximately $144 \mathrm{~mm}$ between the same points respectively.

The TFPRT sensors were chosen from a batch of $100 \Omega\left(R\left(0{ }^{\circ} \mathrm{C}\right)\right)$ commercial type $\mathrm{C} 416$ sensors manufactured by Heraeus Sensor Technology ${ }^{1}$ [2] and conforming to IEC 60751, class B [3]. This model was chosen based on communications with Heraeus engineers which indicated that the type C416 was functionally equivalent to an earlier Heraeus type from circa 2004 then identified as 'M-FX420'. That type TFPRT was successfully used by a group at the National Physical Laboratory (NPL) for satellite black body thermometry in 2004 [4]. In that work, an Ice Melting Point (IMP, $273.15 \mathrm{~K}$ ) reproducibility of $\pm 5 \mathrm{mK}$ (limits for 8 out of 8 specimens) was reported under thermal cycling between $-75^{\circ} \mathrm{C}$ and $130{ }^{\circ} \mathrm{C}$.

Prequalification tests were run at JILA on a sample size of 11 of the type C416 TFPRTs by determining IMP reproducibility under thermal cycling between $0{ }^{\circ} \mathrm{C}$ and $200{ }^{\circ} \mathrm{C}$. Of those eight,

\footnotetext{
${ }^{1}$ Certain commercial equipment, instruments, or materials are identified in this paper in order to specify the experimental procedure adequately. Such identification is not intended to imply recommendation or endorsement by the National Institute of Standards and Technology, nor is it intended to imply that the materials or equipment identified are necessarily the best available for the purpose.
} 
six exhibited reproducibility within limits of $\pm 20 \mathrm{mK}$, two of those exhibited reproducibility of $\pm 6 \mathrm{mK}$, one exhibited reproducibility of $\pm 2 \mathrm{mK}$, and one exhibited reproducibility of $\pm 0.5 \mathrm{mK}$. The most reproducible type C416s were identified as sensors \#1 and \#3. The $\pm 0.5 \mathrm{mK}$ reproducibility TFPRT was mounted on the short probe (described above), and the $\pm 2 \mathrm{mK}$ reproducibility TFPRT was mounted on the long probe. We refer to these two TFPRTs as the 'primary sensors' with indices $n=13,14$. Other sensors, to be described later, are designated as 'base sensors' and were also calibrated for use with these probes. Table 1 lists the basic identifying information for each of the two probes as calibrated at NIST Gaithersburg.

Table 1. Probe and sensor identifying information.

\begin{tabular}{|c|c|c|c|c|}
\hline $\begin{array}{l}\text { Probe } \\
\text { index } i\end{array}$ & $\begin{array}{l}\text { JILA Test } \\
\text { Sensor \# }\end{array}$ & Nominal Length & $\begin{array}{l}\text { NIST Primary Sensor } \\
\text { Identification index }(n)\end{array}$ & $\begin{array}{l}\text { NIST Base Sensor } \\
\text { Identification index }(m)\end{array}$ \\
\hline 1 & 1 & $81 \mathrm{~mm}$ & 13 & 15,17 \\
\hline 2 & 3 & $144 \mathrm{~mm}$ & 14 & 16,18 \\
\hline
\end{tabular}

A test chamber was designed and constructed at JILA for the purposes of transport under vacuum and calibrating the two probes at NIST Gaithersburg. The test chamber is shown in Figures $1 \mathrm{a}$ and $1 \mathrm{~b}$. The design was dictated by several requirements: a) provide a reasonably compact volume with a limiting diameter of less than $178 \mathrm{~mm}$ for use in a water comparison bath in Gaithersburg; b) keep the two sensors in close proximity while accommodating the different glass tube lengths; c). provide as deep immersion below the water bath surface as practical; $d$ ) accommodate ultra-high-vacuum (UHV) practice; and e) allow the backfill of partial pressures of heat-exchange gas and pressure gauging. The test chamber was supplied with a Varian type 24 nude ion gauge and a $38 \mathrm{~mm}$ diameter bakeable UHV valve, both terminated with $71 \mathrm{~mm}$ CF flanges. Prior to transport, the test chamber was baked out and pumped down at JILA to a pressure of $3 \times 10^{-10}$ Torr $\left(4 \times 10^{-8} \mathrm{~Pa}\right)$.

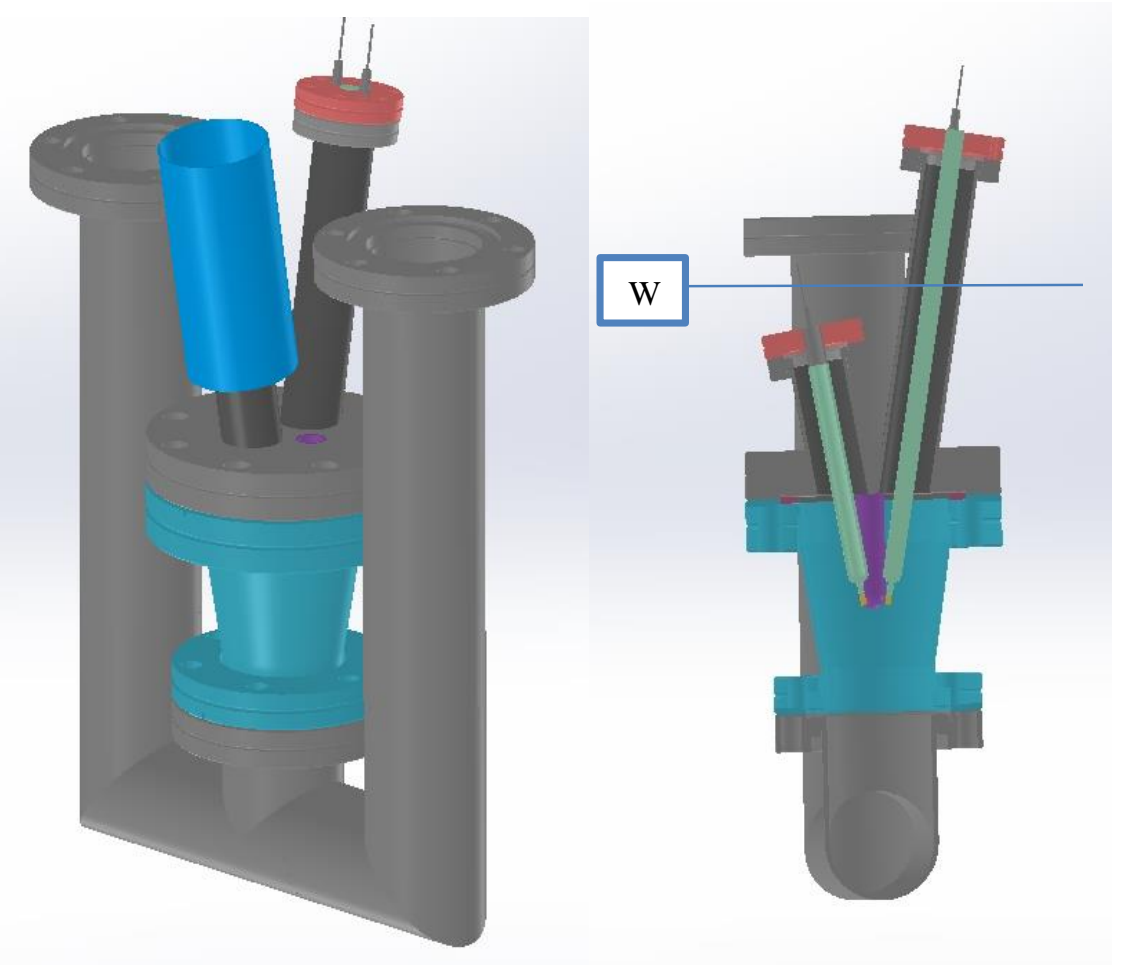


Figure 1. The JILA sensor test chamber. a) showing the water-tight shroud covering the probe 1 base and b) showing the chamber in cross section along the mid-plane including the sensor probes. The approximate location of the bath water line is shown as the horizontal blue line 'W'.

The test chamber was hand carried on a commercial flight and by car, arriving at NIST Gaithersburg on 7-May-2014, and testing began that same day. Prior to opening the UHV valve, the internal pressure was measured using an ion gauge controller borrowed from the NIST Pressure and Vacuum (P\&V) Group, with settings adjusted for the Varian 24 ion gauge. Those readings indicated an internal total pressure of $4 \times 10^{-4}$ Torr $\left(5.3 \times 10^{-2} \mathrm{~Pa}\right)$ at $273.15 \mathrm{~K}$, using either of the two filaments. Based on discussions with NIST P\&V group staff [5], the increase in chamber pressure (presumably from hydrogen outgassing) over approximately two days during transport without pumping was judged to be not unusual for a stainless steel chamber of this size and construction. 


\section{Experimental Descriptions}

\subsection{The NIST Gaithersburg Temperature Calibration Facility}

The NIST Sensor Science Division (SSD), Thermodynamic Metrology Group (TMG) maintains a series of laboratories on the Gaithersburg, MD Campus for thermometer testing, calibration, and research. The JILA SrII sensor probes were calibrated in the TMG's industrial thermometer calibration laboratory, located in building 221, room A242. This laboratory is equipped with Standard Platinum Resistance Thermometers (SPRTs), various precision controlled comparison baths, resistance and voltage instrumentation, and associated standards [6].

The primary sensors were calibrated as received in the test chamber by comparison with a NIST reference SPRT and a check SPRT in a water comparison bath. Two SPRTs were used to determine the bath temperature. The reference thermometer, serial number 4386, and check thermometer, serial number 4388, have both been calibrated according to ITS-90 definitions [7] in the TMG's SPRT calibration laboratory [8]. The water comparison bath is a special deep-well custom-designed forced-circulation bath built by Hart Scientific for NIST with a 42 L working volume. It is similar to a current commercial design, model 7007 sold by Fluke Calibration. This bath can maintain stable temperatures within $\pm 2 \mathrm{mK}$ and non-uniformity $<2 \mathrm{mK}$.

The SPRTs and all test sensor resistances were determined using a MicroK 70 precision thermometry bridge built by Isothermal Technology Ltd $[9,10]$, excitation currents were variable from $0.2 \mathrm{~mA}$ to $0.707 \mathrm{~mA}$ for the primary sensors and $0.5 \mathrm{~mA}$ to $1.0 \mathrm{~mA}$ for the base sensors. The resistance standard was a $100 \Omega$ Tinsley model 5685A, serial number 280167. This standard was calibrated against other standards maintained by the TMG which in turn have been calibrated by the NIST Quantum Measurement Division traceable to the Quantum Hall Ohm.

\subsection{Procedures}

The primary calibration/test period started on May 72014 and ended on May 29, 2014. The experimental test conditions underwent adjustments during the test period and the corresponding testing activities are divided into three segments identified as 'Series 1', 'Series 2', and 'Series 3'. After return of the test chamber to JILA, a separate calibration process was performed to calibrate two TFPRT base sensors (Series 4) from May 302014 to June 5 2014. Another supplemental calibration was performed much later, in October 2014, to calibrate a pair of wirewound PRTs (Series 5) in order to replace those TFPRT base sensors. The experimental uncertainties shown from this point forward are standard $(k=1)$ values.

\subsubsection{Series 1}

Measurements performed prior to opening the test chamber UHV valve and the final installation of the base sensors are referred to as 'Series 1'. No useful data were obtained from this series of measurements since the pressures were too high and the axial thermal gradients were not always known.

An attempt was made to perform a direct Ice Melting Point (IMP) realization by immersion of the test chamber in a crushed ice-water bath. This attempt was abandoned once it was understood that a stable condition could not be achieved due to significant immersion errors which tracked the room temperature variations (see section 3.3). Some measurements were then attempted 
using the water comparison bath at $15{ }^{\circ} \mathrm{C}$, (Figure 2) which showed some improvement in stability, but also showed a significant influence from variations in the laboratory's ambient temperature.

As a temporary modification, to determine the base temperatures, sensors were glued down onto the external surfaces of the $133 \mathrm{CF}$ base flanges of each sensor probe. These 'base sensors' were selected at random from a small collection of Heraeus type C220 class B sensors on hand in Gaithersburg, with no prior calibration. An ex-post facto calibration was performed to improve the base temperature uncertainty ('Series 4', see section 2.2.4).

Prior to opening the valve, the total chamber pressure was again measured using the Varian 24 ion gauge, indicating $6 \times 10^{-4}$ Torr $\left(8 \times 10^{-2} \mathrm{~Pa}\right)$ at $20{ }^{\circ} \mathrm{C}$ on May 9 . Significant heating of the test chamber was observed during use of the Varian 24 ion gauge. It was decided that this gauge could not be used to routinely monitor the chamber pressure and that other ion gauges would be used which were situated farther from the chamber.

\subsubsection{Series 2}

The series 2 measurements were performed from May 12 to 17, after the test chamber vacuum was established and a more permanent installation of the base sensors. The two base sensors were remounted onto cylindrical copper foils designed to fit into inner diameter of the $6.35 \mathrm{~mm}$ OD SS tube extensions of the 133CF flanges at the base of each of the two sensor probes. The corresponding base sensors are identified in Table 1 . In addition, a pair of $3 \mathrm{~mm}$ diameter nylon rods were furnished by JILA with cotton bonded to about half of their lengths and these were

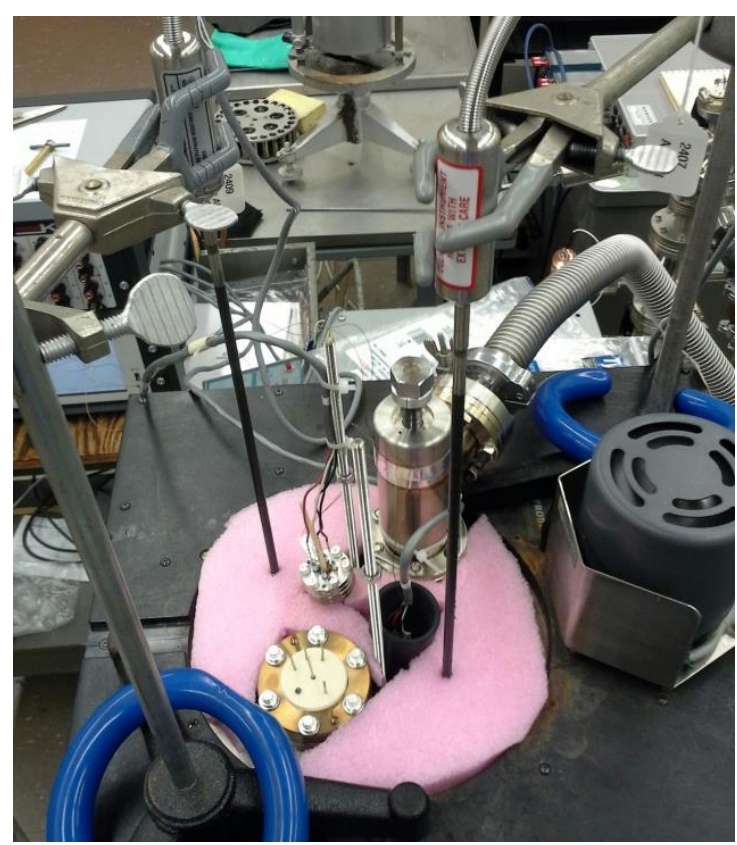

Figure 2. The setup showing the test chamber immersed in the water comparison bath with the SPRTs. inserted into the reentrant portions of the atmosphere side of the glass tubes in each of the probes in order to suppress convection.

A turbomolecular pumping system was connected to the chamber via a $914 \mathrm{~mm}$ length of $25 \mathrm{~mm}$ diameter flexible SS hose and the UHV valve port (see Figure 2). A glass-encapsulated ion gauge located on the pumping system near the inlet of the turbo pump was used for routine readings of the system pressure. The difference between the chamber pressure (Varian 24 Nude Ion Gauge) and the system pressure was determined later under steady-state pumping conditions. The UHV valve was opened for the first time on May 12 and the test chamber was evacuated overnight using the turbo pumping system. The final experimental setup is shown in Figure 2. 
Starting from May 13, all sensor readings corresponding to 'vacuum' conditions would be initiated once the system ion gauge indicated a pressure $\lesssim 1 \times 10^{-7}$ Torr $\left(1.3 \times 10^{-5} \mathrm{~Pa}\right)$. The normal procedure for a set of measurements at a fixed calibration temperature was as follows:

1. Establish the test chamber pressure of $\lesssim 1 \times 10^{-7}$ Torr $\left(1.3 \times 10^{-5} \mathrm{~Pa}\right)$.

2. Log 'vacuum' data for two SPRTs, two primary sensors and two base sensors. SPRTs and base sensor currents were held at fixed excitation current of at $1 \mathrm{~mA}$. Primary sensors were measured at $0.2 \mathrm{~mA}, 0.5 \mathrm{~mA}, 0.707 \mathrm{~mA}$ and then repeating $0.2 \mathrm{~mA}$, corresponding to $4.3 \mu \mathrm{W}, 27 \mu \mathrm{W}$ and $54 \mu \mathrm{W}$, waiting approximately $1 \mathrm{~h}$ for equilibration after each change in sensor current.

3. Introduce an exchange gas of argon or helium into the chamber at an indicated pressure of $\approx 3$ Torr $(400 \mathrm{~Pa})$, then wait $\approx 1 \mathrm{~h}$ for re-equilibration.

4. Again, log 'Ar' or 'He' data for two SPRTs, two primary sensors and two base sensors. Primary sensors were similarly measured at $0.2 \mathrm{~mA}, 0.5 \mathrm{~mA}, 0.707 \mathrm{~mA}$ and then repeating $0.2 \mathrm{~mA}$, corresponding to $4.3 \mu \mathrm{W}, 27 \mu \mathrm{W}$ and $54 \mu \mathrm{W}$, waiting approximately $0.5 \mathrm{~h}$ to $1 \mathrm{~h}$ for equilibration after each change in sensor current.

5. If a single measurement set involved taking data for both argon and helium exchange gas conditions in a single day, the helium backfill was done before the argon and the chamber was re-evacuated between backfills. The reasoning for this was that a small amount of helium contamination in the argon should have relatively little effect of the effective thermal conductivity of the argon, while the converse situation would be more noticeable due to collisions by the helium atoms with the much heavier argon impurities.

6. Re-evacuate the chamber overnight and adjust the bath temperature for the next-day calibration.

The exchange-gas pressure was monitored with a Lesker type 910 wide-range combination Pirani-Piezo pressure gauge using a nominal calibration curve adjusted for $\mathrm{N}_{2}$ gas. Therefore for a nominal indicated gauge reading of 3 Torr $(400 \mathrm{~Pa})$, the actual chamber pressure for argon would be closer to 7 Torr $(930 \mathrm{~Pa})$ and about 2 Torr $(270 \mathrm{~Pa})$ for helium. In either case, these pressures were judged sufficiently high to serve as a basis for strong thermal coupling to the water bath and establishing isothermal conditions inside the chamber at a known temperature (as determined by the SPRTs). A more accurate knowledge of the gas pressures is not necessary. Both the Ar and He gas supply cylinders contained nominally $99.995 \%$ purity gas. In practice there was some evidence of contamination of the gases, particularly the helium with other heavier species, either air or argon. This may have been due to inadequate purge and flush procedures in the gas manifold.

The Series 2 measurements comprised calibration data at nominal temperatures of $20{ }^{\circ} \mathrm{C}, 25^{\circ} \mathrm{C}$, $30{ }^{\circ} \mathrm{C}$, and $35^{\circ} \mathrm{C}$. Data were obtained using both Ar and He exchange gas, except for the $20{ }^{\circ} \mathrm{C}$ point, which only included argon and did not include helium.

\subsubsection{Series 3}

The series 3 measurements were performed from May 23 to 29, at nominal calibration temperatures of $20^{\circ} \mathrm{C}, 22{ }^{\circ} \mathrm{C}, 24^{\circ} \mathrm{C}, 25^{\circ} \mathrm{C}$ and $27^{\circ} \mathrm{C}$. The set up and procedures closely followed those established for the earlier Series 2 measurements, with only two exceptions: 
a) the $0.5 \mathrm{~mA}$ primary sensor current was usually skipped as it was deemed unnecessary; and

b) only helium exchange gas was used.

Other more subtle differences in the Series 3 measurements were improvements in the ambient laboratory temperature stability and additional insulation used at the top opening to the water bath. In the former case, access to the laboratory was restricted and the overhead lights were kept off whenever measurements were underway (May 27-29), keeping the ambient conditions generally within $\pm 0.1^{\circ} \mathrm{C}$ during the course of a day. In the latter case, the improved insulation would have reduced the vertical gradients near the surface of the water bath, but there are no measurements to verify if any reduction was achieved.

\subsubsection{Series 4}

The series 4 measurements were supplemental to the primary sensor calibrations and undertaken only to perform a simple calibration on the two TFPRT base sensors, identified with indices $m=15,16$. Calibration data between $0{ }^{\circ} \mathrm{C}$ and $45^{\circ} \mathrm{C}$ were obtained from May 30 to June 6 using the same equipment as used in the earlier measurement series, except that the base sensors were mounted into $8 \mathrm{~mm}$ OD glass test tubes for water bath and ice bath immersion.

\subsubsection{Series 5}

An additional series of supplemental measurements were made to calibrate an extra pair of base sensors identified by indices $m=17,18$. These sensors were $100 \Omega$ wire-wound (WW) PRTs made from high-purity Pt wire which conformed to a different specification [11] than the TF types. The calibration data for this series were obtained from 15 to 17 October over the range $0.01{ }^{\circ} \mathrm{C}$ to $35^{\circ} \mathrm{C}$ in Building 221 , Room B217 on the Gaithersburg campus using different equipment from the earlier series of measurements.

All measurements in this series were made using a miniature $11 \mathrm{~mm}$ OD aluminum comparison block within a close-fitting $13 \mathrm{~mm}$ OD glass tube. Both WW PRTs were co-located inside the aluminum block along with a $25.5 \Omega$ capsule-type SPRT, s/n 3368, calibrated on the ITS-90. The tube was immersed inside the well of a WTP cell for measurements at $0.01{ }^{\circ} \mathrm{C}$ and immersed within an actively-controlled stirred-oil bath for the comparison measurements from $15{ }^{\circ} \mathrm{C}$ to $35^{\circ} \mathrm{C}$. All resistance readings were made using an $\mathrm{AC}$ resistance bridge using a $30 \mathrm{~Hz}$ carrier relative to a calibrated standard resistor maintained at $25^{\circ} \mathrm{C}$. Measurement currents were varied between $1 \mathrm{~mA}$ and $2 \mathrm{~mA}$ and resistances extrapolated to $0 \mathrm{~mA}$ for calibration purposes. 


\section{Calibration Results}

\subsection{Primary Sensors}

The first feature of importance in the data is the variable self-heating of the sensors, which varied (qualitatively) as expected according to the test chamber pressure conditions over the Joule heating rates from $4 \mu \mathrm{W}$ to $57 \mu \mathrm{W}$. Figure 3 shows self-heating data obtained at $25^{\circ} \mathrm{C}$ for vacuum, 2 Torr $\mathrm{He}(270 \mathrm{~Pa})$ and 7 Torr $(930 \mathrm{~Pa}) \mathrm{Ar}$.

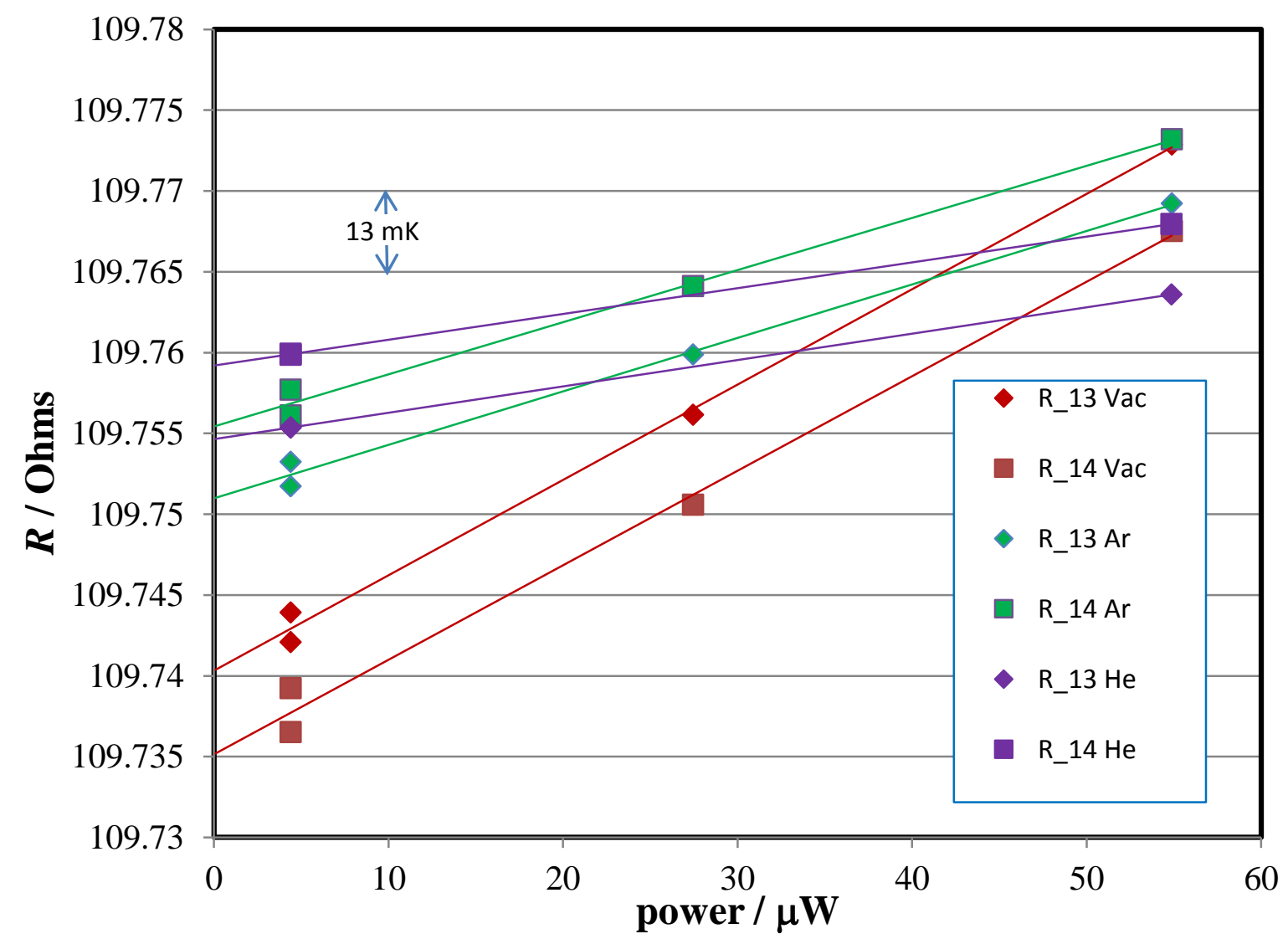

Figure 3. Self-heating plots for both primary sensors at $25^{\circ} \mathrm{C}$ under 2 Torr $(270 \mathrm{~Pa}) \mathrm{He}, 7$ Torr $(930 \mathrm{~Pa})$ Ar, and vacuum $\left(<1 \times 10^{-7}\right.$ Torr $\left.\left(1.3 \times 10^{-5} \mathrm{~Pa}\right)\right)$ from Series 2 .

All primary sensor calibration data are extrapolated to zero-power resistance using the selfheating curves like the one shown in Figure 3. The fact that there is a significant offset between the zero power resistance under vacuum and under exchange gas conditions is an indication of the influence of ambient temperature as a thermal perturbation under vacuum conditions. In fact it was found that this offset between vacuum and helium conditions varied linearly with the difference between ambient temperature and the calibration bath temperature, and also in proportion to the base temperature-bath temperature difference (i.e. the 'axial gradient'). This is because the base flange temperatures, as indicated by the base sensors, would always passively equilibrate to a temperature intermediate between the bath temperature and ambient temperature. In either case, the effect can be explained as a normal consequence of imperfect immersion and will be discussed in more detail below. 
The reproducibility of the $0.2 \mathrm{~mA}(\approx 4.5 \mu \mathrm{W}$ power) vacuum data were limited by drift in the ambient laboratory temperature during the measurements due to the immersion error offsets. These offsets have been correlated with the axial gradients for each probe and are treated below (section 3.3) and in section 4.1.2. In contrast, the helium data reproducibility was limited only by the bath temperature stability and showed no dependence on ambient temperature drift or fluctuations. The argon data were less reproducible than the helium data and appeared to be limited by both water bath temperature and ambient temperature fluctuations. This observation led to the discontinuation of argon exchange gas after series 2 was completed in favor of exclusive use of helium thereafter.

The slopes of the self-heating curves were remarkably similar between the two probes for constant conditions in the test chamber. These slopes were also reasonably independent of bath temperature, ambient temperature, and axial gradients. A conventional term for the slope is the 'self-heating coefficient', $\eta$, which is equivalent to an effective thermal resistance for a heat transfer network of series and parallel resistances.

Table 2 provides a summary of the zero-power resistance calibration data for the primary sensors $\# 13$ and \#14. Table 3 lists a summary of the associated $1 \mathrm{~mA}$ resistance data for the base sensors $\# 15$ and \#16. The base sensor data are averages taken over the same time periods as the vacuum data for the primary sensors. Table 4 is a summary of the self-heating data associated with the two primary sensors, including $\eta$ values for all vacuum, argon and helium data. In all cases the standard uncertainty $s$ is derived from the normal statistical variance alone.

The helium data were used as the basis for the calibrations of the two primary sensors. A 3parameter mild-quadratic function $R(t)$ for resistance as a function of temperature $t\left(\right.$ in ${ }^{\circ} \mathrm{C}$ ) is used to fit the data and then re-parameterized equivalent to the IEC industrial PRT specification [3],

$$
R(t)=R_{0}\left(1+A t+B t^{2}\right)
$$

Since $R_{0}$ can be determined with much greater relative precision than $A$ and $B$, there is no real loss in precision by factoring out $R_{0}$ from the other two coefficients.

Table 5a lists the calibration fit parameters and associated uncertainties for the two primary sensors when fitting all three parameters in Eqn. 1. When these estimated standard errors are used as uncertainties, however, they grossly overestimate the interpolation uncertainty when using Eqn. 1 or its inverse to calculate temperature. The normal procedure for PRT calibration is to obtain $R_{0}$ independently as a fixed point realization, use it to reparameterize the data as ratios $W \equiv R(t) / R_{0}$, and then fit $W$-1 for the remaining parameters. This procedure results in much lower estimated errors in $A$ and $B$, but it is not available in this case since IMP realizations were found to be impractical using the test chamber. The solution is to take the estimated $R_{0}$ values from the first unconstrained fits of $R(t)$, and then refit the data in $W$-1 constrained so that the intercept $W(0)-1=0$. The resulting estimated coefficients are shown in Table $5 \mathrm{~b}$. The $A$ and $B$ coefficients are essentially equivalent to their previous estimates (well within the new smaller error estimates), but with much lower estimated errors, and also nearly equivalent between the two sensors. This is an acceptable practice as long as $R_{0}$ is not intended to provide interpolation near $0{ }^{\circ} \mathrm{C}$. Rather, in this case its role is that of a scaling parameter for the resistance unit and hence is 
known to a much lower uncertainty than the $\approx 50 \mu \Omega / \Omega$ given in Table 5 a. (see section 4.1 .4 under Discussion)

Figure 4 shows the deviation of the primary sensors' calibrations with respect to the IEC nominal specification in terms of a temperature-equivalent difference in resistance. The primary sensors would qualify as IEC class AA tolerance over this limited range despite the fact that the sensors are categorized as Class $\mathrm{B}$ (Class $\mathrm{B}=3 \times$ Class $\mathrm{AA}$ ). These deviations from the nominal IEC curve range from $41 \mathrm{mK}$ to $64 \mathrm{mK}$ and are highly correlated between the two sensors. A closer look at the degree of correlation in the sensor data is shown in Figure 5 which is a plot of the residuals for both sensor fits to Eqn. 1. The correlation coefficient for the two sets of residuals is 0.99989 and the residuals are all within $\pm 1.2 \mathrm{mK}$. The origin of the correlation is most likely due to temperature transients and or spatial gradients in the water bath which are in phase for the two primary sensors. Both sets of coefficients, either from Table 5a or Table 5b, yield practically identical residuals. 
Table 2. Summary of zero-power resistance data for the primary sensors \# 13 and \# 14 . All resistances are in Ohms, all temperatures are in ${ }^{\circ} \mathrm{C}$.

\begin{tabular}{|c|c|c|c|c|c|c|c|c|c|c|c|c|c|c|c|}
\hline Date & Ser. & $T_{\text {bath }}$ & $s\left(T_{\text {bath }}\right)$ & $R_{13 \text { _vac }}$ & $s\left(R_{13 \_v a c}\right)$ & $R_{13 \_\mathrm{Ar}}$ & $s\left(R_{13 \_\mathrm{Ar}}\right)$ & $R_{13 \_ \text {He }}$ & $s\left(R_{13 \_\mathrm{He}}\right)$ & $R_{14 \_v a c}$ & $s\left(R_{14 \_ \text {vac }}\right)$ & $R_{14 \_\mathrm{Ar}}$ & $s\left(R_{14 \_\mathrm{Ar}}\right)$ & $R_{14 \_\mathrm{He}}$ & $s\left(R_{14 \_\mathrm{He}}\right)$ \\
\hline $13 / 05 / 14$ & 2 & 20.0229 & 0.0013 & 107.8388 & 0.0007 & 107.8226 & 0.0001 & & & 107.8553 & 0.0012 & 107.8273 & 0.0001 & & \\
\hline $26 / 05 / 14$ & 3 & 20.0233 & 0.0012 & 107.8354 & 0.0007 & & & 107.8189 & 0.0002 & 107.8468 & 0.0010 & & & 107.8234 & 0.0003 \\
\hline $27 / 05 / 14$ & 3 & 22.0019 & 0.0004 & 108.5922 & 0.0009 & & & 108.5869 & 0.00004 & 108.5975 & 0.0010 & & & 108.5915 & 0.00003 \\
\hline $28 / 05 / 14$ & 3 & 23.9658 & 0.0005 & 109.3439 & 0.0002 & & & 109.3500 & 0.0001 & 109.3436 & 0.0003 & & & 109.3545 & 0.0001 \\
\hline $28 / 05 / 14$ & 3 & 25.0101 & 0.0005 & 109.7458 & 0.0001 & & & 109.7554 & 0.00002 & 109.7434 & 0.0001 & & & 109.7599 & 0.00001 \\
\hline $14 / 05 / 14$ & 2 & 25.0082 & 0.0010 & 109.7403 & 0.0007 & 109.7510 & 0.0006 & & & 109.7351 & 0.0011 & 109.7554 & 0.0006 & & \\
\hline $17 / 05 / 14$ & 2 & 25.0091 & 0.0015 & & & & & 109.7546 & 0.00001 & & & & & 109.7592 & 0.00003 \\
\hline $29 / 05 / 14$ & 3 & 26.9836 & 0.0003 & 110.5059 & 0.0001 & & & 110.5218 & 0.00003 & 110.4999 & 0.0001 & & & 110.5263 & 0.00004 \\
\hline $15 / 05 / 14$ & 2 & 30.0161 & 0.0008 & 111.6513 & 0.0006 & 111.6891 & 0.0002 & 111.6981 & 0.00003 & 111.6290 & 0.0010 & 111.6935 & 0.0002 & 111.7026 & 0.00002 \\
\hline $16 / 05 / 14$ & 2 & 35.0253 & 0.0004 & 113.5544 & 0.0017 & 113.622 & 0.0002 & 113.6384 & 0.00016 & 113.5149 & 0.0001 & 113.6265 & 0.00025 & 113.6428 & 0.00017 \\
\hline
\end{tabular}

Table 3. Summary of $1 \mathrm{~mA}$ resistance and temperature data for the base sensors, \#15 and \#16. All resistances are in Ohms, and temperatures in ${ }^{\circ} \mathrm{C}$.

\begin{tabular}{|l|l|l|l|l|l|c|c|c|l|}
\hline Date & Ser. & $R_{15}$ & $s\left(R_{15}\right)$ & $T_{15}$ & $s\left(T_{15}\right)$ & $R_{16}$ & $s\left(R_{16}\right)$ & $T_{16}$ & \multicolumn{1}{|c|}{$s\left(T_{16}\right)$} \\
\hline $13 / 05 / 2014$ & 2 & 107.8240 & 0.0013 & 20.1829 & 0.0035 & 108.5508 & 0.0196 & 21.9257 & 0.050 \\
\hline $26 / 05 / 2014$ & 3 & 107.8180 & 0.0017 & 20.1673 & 0.0043 & 108.2655 & 0.0171 & 21.1888 & 0.044 \\
\hline $27 / 05 / 2014$ & 3 & 108.5549 & 0.0018 & 22.0684 & 0.0047 & 108.6703 & 0.0196 & 22.2344 & 0.051 \\
\hline $28 / 05 / 2014$ & 3 & 109.2854 & 0.0004 & 23.9543 & 0.0010 & 109.0814 & 0.0033 & 23.2964 & 0.0085 \\
\hline $28 / 05 / 2014$ & 3 & 109.6796 & 0.0004 & 24.9725 & 0.0009 & 109.3498 & 0.0019 & 23.9900 & 0.0048 \\
\hline $14 / 05 / 2014$ & 2 & 109.6690 & 0.0018 & 24.9452 & 0.0046 & 109.2135 & 0.0241 & 23.6378 & 0.063 \\
\hline $29 / 05 / 2014$ & 3 & 110.4258 & 0.0004 & 26.9016 & 0.0012 & 109.8873 & 0.0035 & 25.3796 & 0.0090 \\
\hline $15 / 05 / 2014$ & 2 & 111.4955 & 0.0030 & 29.6694 & 0.0078 & 109.9381 & 0.0206 & 25.5109 & 0.054 \\
\hline $16 / 05 / 2014$ & 2 & 113.2703 & 0.0261 & 34.2687 & 0.0679 & 110.4941 & 0.070 & 26.9489 & 0.18 \\
\hline
\end{tabular}


Table 4. Summary of the self-heating coefficients $\eta$ for the two primary sensors \#13 and \#14. The units for $\eta$ are $\mathrm{mK} / \mu \mathrm{W}$ and ${ }^{\circ} \mathrm{C}$ for temperature.

\begin{tabular}{|c|c|c|c|c|c|c|c|c|c|c|c|c|c|c|c|}
\hline Date & Ser. & $T_{\text {bath }}$ & $s\left(T_{\text {bath }}\right)$ & $\eta_{13 \mathrm{vac}}$ & $s\left(\eta_{13 \mathrm{vac}}\right)$ & $\eta_{13 \_A r}$ & $s\left(\eta_{13 \_A r}\right)$ & $\eta_{13 \_ \text {He }}$ & $s\left(\eta_{13 \_ \text {He }}\right)$ & $\eta_{14 \mathrm{vac}}$ & $s\left(\eta_{14 \mathrm{vac}}\right)$ & $\eta_{14 \_A r}$ & $s\left(\eta_{14 \_A r}\right)$ & $\eta_{14 \_ \text {He }}$ & $s\left(\eta_{14 \_\mathrm{He}}\right)$ \\
\hline $13 / 05 / 2014$ & 2 & 20.0229 & 0.0013 & 1.61 & 0.06 & 0.90 & 0.01 & & & 1.60 & 0.10 & 0.88 & 0.01 & & \\
\hline $26 / 05 / 2014$ & 3 & 20.0233 & 0.0012 & 1.51 & 0.06 & & & 0.655 & 0.020 & 1.47 & 0.08 & & & 0.638 & 0.022 \\
\hline $27 / 05 / 2014$ & 3 & 22.0019 & 0.0004 & 1.48 & 0.07 & & & 0.451 & 0.003 & 1.45 & 0.08 & & & 0.442 & 0.003 \\
\hline $28 / 05 / 2014$ & 3 & 23.9658 & 0.0005 & 1.49 & 0.01 & & & 0.462 & 0.011 & 1.45 & 0.02 & & & 0.452 & 0.010 \\
\hline $28 / 05 / 2014$ & 3 & 25.0101 & 0.0005 & 1.47 & 0.01 & & & 0.479 & 0.002 & 1.42 & 0.01 & & & 0.469 & 0.001 \\
\hline $14 / 05 / 2014$ & 2 & 25.0082 & 0.0010 & 1.54 & 0.06 & 0.86 & 0.05 & & & 1.52 & 0.09 & 0.84 & 0.05 & & \\
\hline $17 / 05 / 2014$ & 2 & 25.0091 & 0.0015 & & & & & 0.424 & 0.001 & & & & & 0.416 & 0.002 \\
\hline $29 / 05 / 2014$ & 3 & 26.9836 & 0.0003 & 1.46 & 0.01 & & & 0.494 & 0.002 & 1.43 & 0.01 & & & 0.482 & 0.003 \\
\hline $15 / 05 / 2014$ & 2 & 30.0161 & 0.0008 & 1.48 & 0.05 & 0.85 & 0.01 & 0.410 & 0.002 & 1.45 & 0.08 & 0.83 & 0.01 & 0.401 & 0.002 \\
\hline $16 / 05 / 2014$ & 2 & 35.0253 & 0.0004 & 1.46 & 0.14 & 0.83 & 0.02 & 0.425 & 0.012 & 1.53 & 0.01 & 0.81 & 0.02 & 0.417 & 0.013 \\
\hline
\end{tabular}

Table 5a. Helium calibration parameters for the primary sensors according to Equation 1 using zero-power resistances and fitting all three parameters.

\begin{tabular}{|l|c|c|c|c|}
\hline parameter & $\# 13$ Value & $\# 13$ Std. Error & $\# 14$ Value & $\# 14$ Std. Error \\
\hline$R_{0} / \Omega$ & 100.0164 & 0.0052 & 100.0207 & 0.0053 \\
\hline$A /{ }^{\circ} \mathrm{C}^{-1}$ & 0.0039055 & $3.9 \mathrm{E}-06$ & 0.0039055 & $3.9 \mathrm{E}-06$ \\
\hline$B /{ }^{\circ} \mathrm{C}^{-2}$ & $-4.82 \mathrm{E}-07$ & $7.0 \mathrm{E}-08$ & $-4.86 \mathrm{E}-07$ & $7.0 \mathrm{E}-08$ \\
\hline
\end{tabular}

Table 5b. Helium calibration parameters for the primary sensors using $W(t)-1$ and zero-power resistances fitting $A \& B$ parameters only.

\begin{tabular}{|l|c|c|c|c|}
\hline parameter & $\# 13$ Value & $\# 13$ Std. Error & $\# 14$ Value & $\# 14$ Std. Error \\
\hline$R_{0} / \Omega$ & 100.0164 & - & 100.0207 & - \\
\hline$A /{ }^{\circ} \mathrm{C}^{-1}$ & 0.0039054 & $3.0 \mathrm{E}-07$ & 0.0039055 & $3.1 \mathrm{E}-07$ \\
\hline$B /{ }^{\circ} \mathrm{C}^{-2}$ & $-4.82 \mathrm{E}-07$ & $1.1 \mathrm{E}-08$ & $-4.86 \mathrm{E}-07$ & $1.1 \mathrm{E}-08$ \\
\hline
\end{tabular}




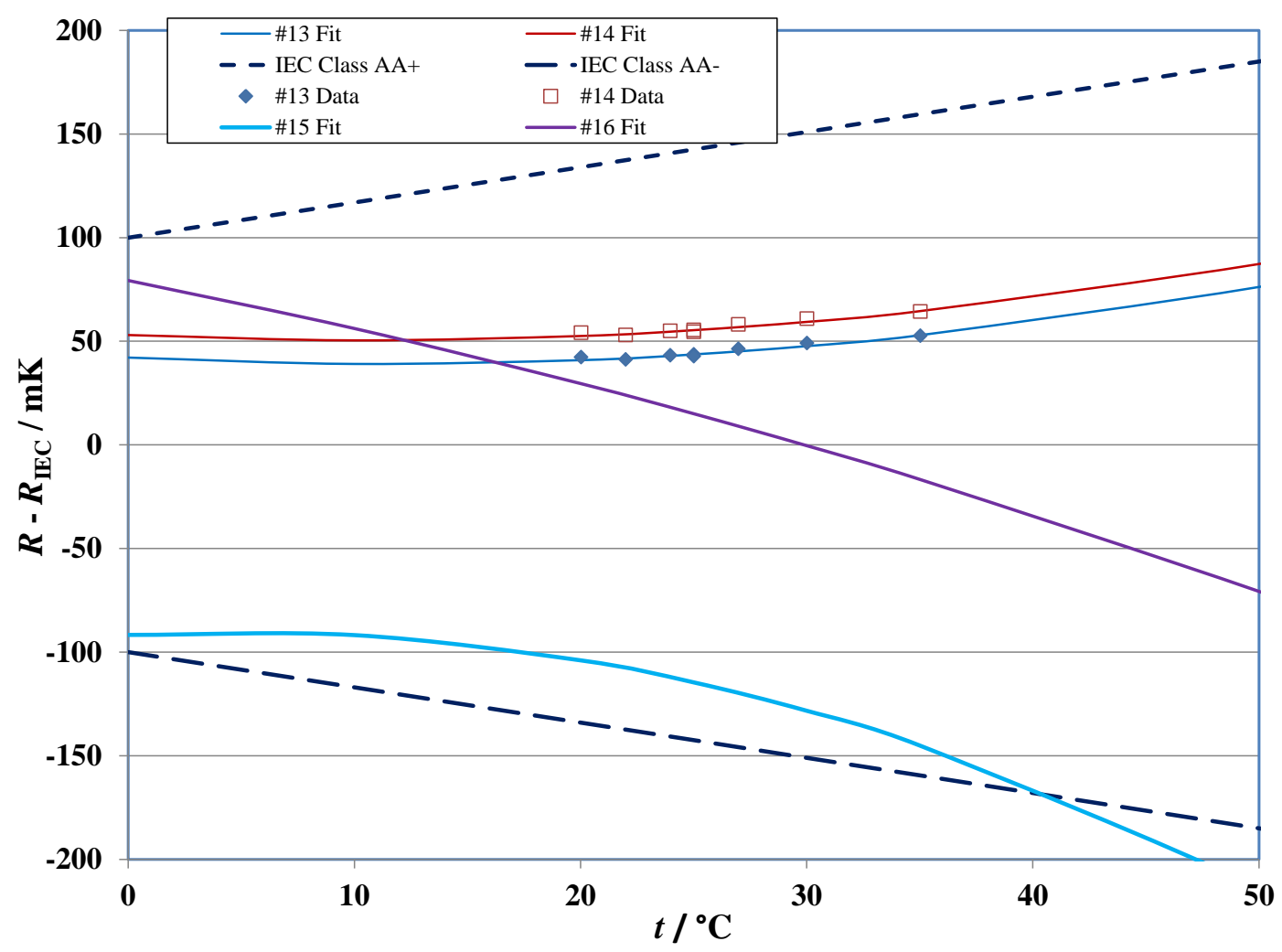

Figure 4. Deviations of the primary and base sensor resistance calibration data and curve fits from the nominal IEC curve for a Pt100 thermometer. IEC class AA tolerance bands are shown for comparison.

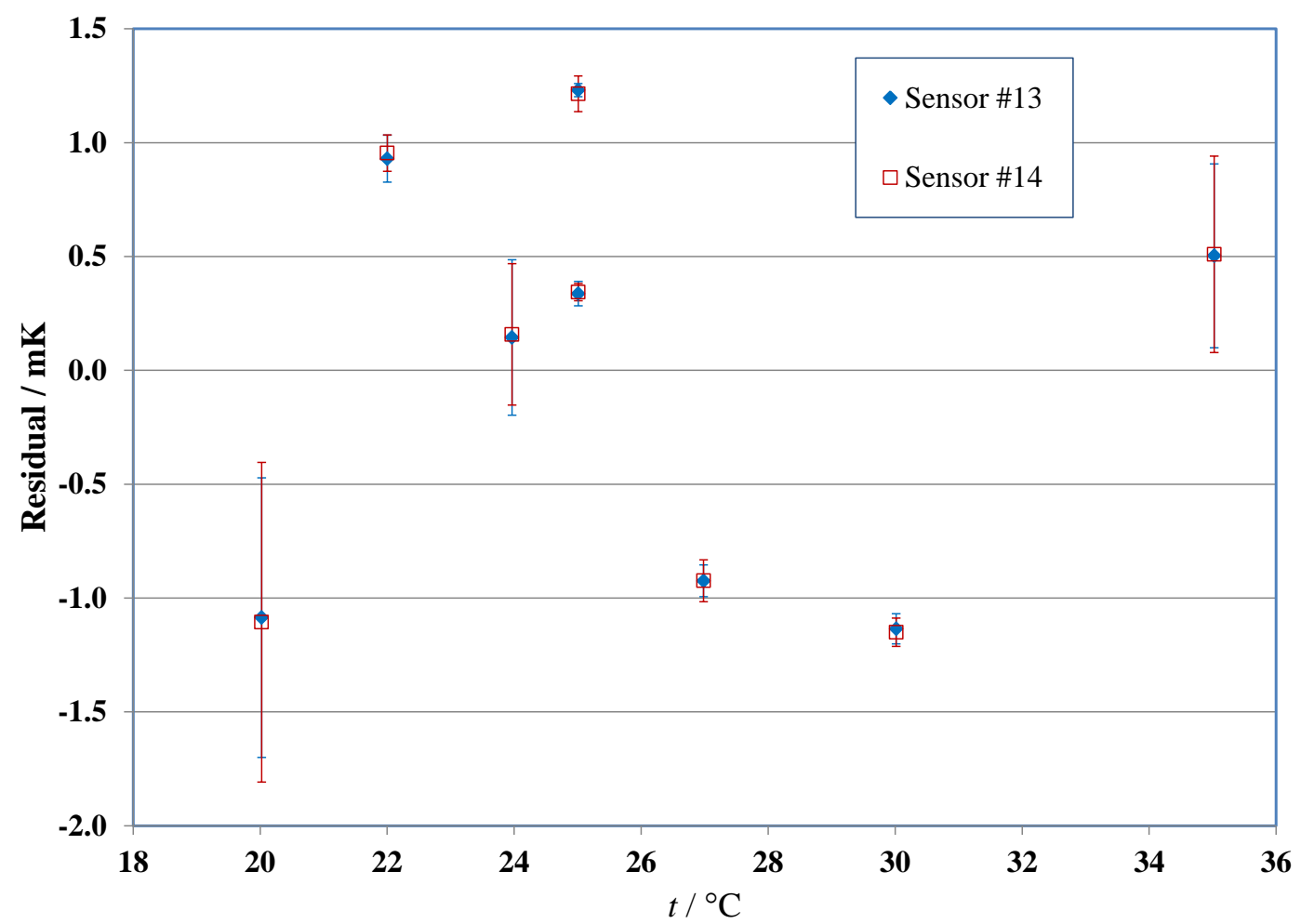

Figure 5. Fit residuals to Eqn. 1 for the two primary sensors. The error bars represent standard statistical (i.e. nominally random) uncertainty only. 


\subsection{Base Sensors}

\subsubsection{Thin Film Types}

Base sensor calibration data for the \# 15 and \#16 TFPRTs were obtained between $0{ }^{\circ} \mathrm{C}$ and $45^{\circ} \mathrm{C}$; however, the IMP data $\left(0{ }^{\circ} \mathrm{C}\right)$ was noticeably less reproducible than the other data taken at higher temperatures in the water comparison bath. Transient stress-relaxation effects were observed in most of the IMP data, particularly for sensor \#16. This behavior was unusual, and necessitated discarding the IMP data and fitting the $R_{0}$ parameter in Eqn. 1 indirectly using calibration data at $14{ }^{\circ} \mathrm{C}, 25^{\circ} \mathrm{C}, 35^{\circ} \mathrm{C}$ and $45^{\circ} \mathrm{C}$. This decision should be acceptable given that the base sensors will not likely be used at temperatures lower than $16^{\circ} \mathrm{C}$. Tables $6 \mathrm{a}$ and $6 \mathrm{~b}$ list the $1 \mathrm{~mA}$ and $0 \mathrm{~mA}$ calibration coefficients, respectively, for the two base sensors and associated statistical uncertainties. The $1 \mathrm{~mA}$ calibration curves for the base sensors are also shown in Figure 4 relative to the nominal IEC curve.

Table 6a. $1 \mathrm{~mA}$ Calibration coefficients for the two TF base sensors.

\begin{tabular}{|c|c|c|c|c|}
\hline parameter & $\# 15$ Value & $\# 15$ Std. Error & $\# 16$ Value & $\# 16$ Std. Error \\
\hline$R_{0} / \Omega$ & 99.9642 & 0.0039 & 100.0310 & 0.0035 \\
\hline$A /{ }^{\circ} \mathrm{C}^{-1}$ & 0.0039121 & $2.8 \mathrm{E}-06$ & 0.0038986 & $2.5 \mathrm{E}-06$ \\
\hline$B /{ }^{\circ} \mathrm{C}^{-2}$ & $-8.12 \mathrm{E}-07$ & $4.6 \mathrm{E}-08$ & $-6.41 \mathrm{E}-07$ & $4.2 \mathrm{E}-08$ \\
\hline
\end{tabular}

Table 6b. $0 \mathrm{~mA}$ Calibration coefficients for the two TF base sensors.

\begin{tabular}{|c|l|c|l|c|}
\hline parameter & $\# 15$ Value & $\# 15$ Std. Error & $\# 16$ Value & $\# 16$ Std. Error \\
\hline$R_{0} / \Omega$ & 99.9575 & 0.0044 & 100.0267 & 0.0018 \\
\hline$A /{ }^{\circ} \mathrm{C}^{-1}$ & 0.0039122 & $3.2 \mathrm{E}-06$ & 0.0038979 & $1.3 \mathrm{E}-06$ \\
\hline$B /{ }^{\circ} \mathrm{C}^{-2}$ & $-8.09 \mathrm{E}-07$ & $5.3 \mathrm{E}-08$ & $-6.25 \mathrm{E}-07$ & $2.2 \mathrm{E}-08$ \\
\hline
\end{tabular}

The $1 \mathrm{~mA}$ fit residuals for the two base sensors are shown in Figure 6. In addition to the calibration data at $14{ }^{\circ} \mathrm{C}, 25^{\circ} \mathrm{C}, 35^{\circ} \mathrm{C}$ and $45^{\circ} \mathrm{C}$, two other sets of residuals are shown at $27^{\circ} \mathrm{C}$ and $40^{\circ} \mathrm{C}$. These points were excluded from the $1 \mathrm{~mA}$ fits because they were obtained in earlier comparisons, before apparent resistance shifts and stress-relaxation effects were observed at $0{ }^{\circ} \mathrm{C}$. All the residuals including the extra points are included in Figure 6 to help estimate the stability of the base sensors. 


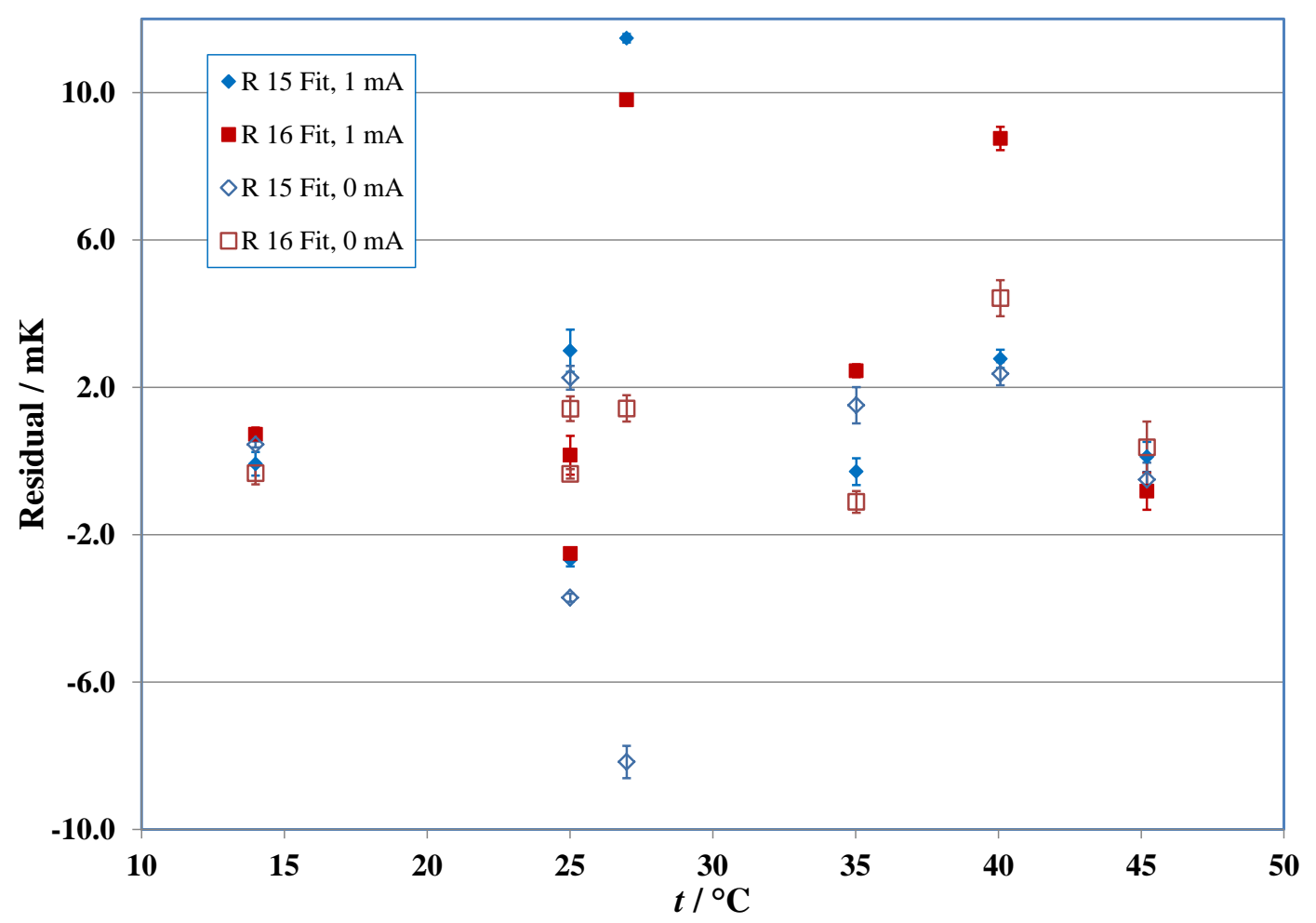

Figure 6a. Fit residuals to Eqn. 1 for the two TF base sensors $m=15,16$ for both $1 \mathrm{~mA}$ and $0 \mathrm{~mA}$ data. The error bars represent standard statistical (i.e. nominally random) uncertainty only. The $27^{\circ} \mathrm{C}$ and $40{ }^{\circ} \mathrm{C}$ data were excluded from the $1 \mathrm{~mA}$ fit.

\subsubsection{Wire Wound Types}

Base sensor calibration data for the \# 17 and \#18 WW PRTs were obtained between $0.01{ }^{\circ} \mathrm{C}$ and $35^{\circ} \mathrm{C}$. Water Triple-point resistance data $\left(0.01{ }^{\circ} \mathrm{C}\right)$ were obtained in place of the customary IMP data $\left(0{ }^{\circ} \mathrm{C}\right)$ at the beginning and end of the calibration sequence. For the purposes of interpolation via Eqn. 1, the normal interpretation of the $R_{0}$ parameter was retained by making a simple conversion given by $R_{0}=0.999961 R_{\text {wtp }}$ which is correct for all platinum of this grade. The calibration coefficients where then obtained in the conventional way by fitting Eqn. 1 in the form of $W-1$ versus $t$ in ${ }^{\circ} \mathrm{C}$ for all the data in the range. These included nominal calibration points at $0.01{ }^{\circ} \mathrm{C}, 15^{\circ} \mathrm{C}, 20{ }^{\circ} \mathrm{C}, 25{ }^{\circ} \mathrm{C}, 30^{\circ} \mathrm{C}$, and $35^{\circ} \mathrm{C}$. The results are shown in Tables 7 for the $0 \mathrm{~mA}$ data.

Table 7. The $0 \mathrm{~mA}$ Calibration coefficients for the two WW base sensors.

\begin{tabular}{|c|c|c|c|c|}
\hline parameter & $\# 17$ Value & $\# 17$ Std. Error & $\# 18$ Value & $\# 18$ Std. Error \\
\hline$R_{0} / \Omega$ & 99.97467 & 0.00011 & 100.0271 & 0.00015 \\
\hline$A /{ }^{\circ} \mathrm{C}^{-1}$ & $3.98656 \mathrm{E}-03$ & $1.9 \mathrm{E}-07$ & $3.98508 \mathrm{E}-03$ & $1.6 \mathrm{E}-07$ \\
\hline$B /{ }^{\circ} \mathrm{C}^{-2}$ & $-6.085 \mathrm{E}-07$ & $6.8 \mathrm{E}-09$ & $-6.112 \mathrm{E}-07$ & $5.6 \mathrm{E}-09$ \\
\hline
\end{tabular}

The \#17 and \#18 WW base sensors exhibited superior reproducibility compared to the TF types $\# 15$ and \#16. This resulted in much lower uncertainties in the fit parameters as shown in Table 7, approximately a factor of 10 lower in $u\left(R_{0}\right), 17 \times$ lower in $u(A)$ and $8 \times$ lower in $u(B)$ or more 
compared to those for the TF base sensors. The fit residuals as shown in Figure $6 \mathrm{~b}$ are similarly much lower for the WW base sensors compared to the TF types. With only a few exceptions, the residuals for all the data are within the limits of the statistical (i.e. random) uncertainties alone, typically $\pm 1.5 \mathrm{mK}$.

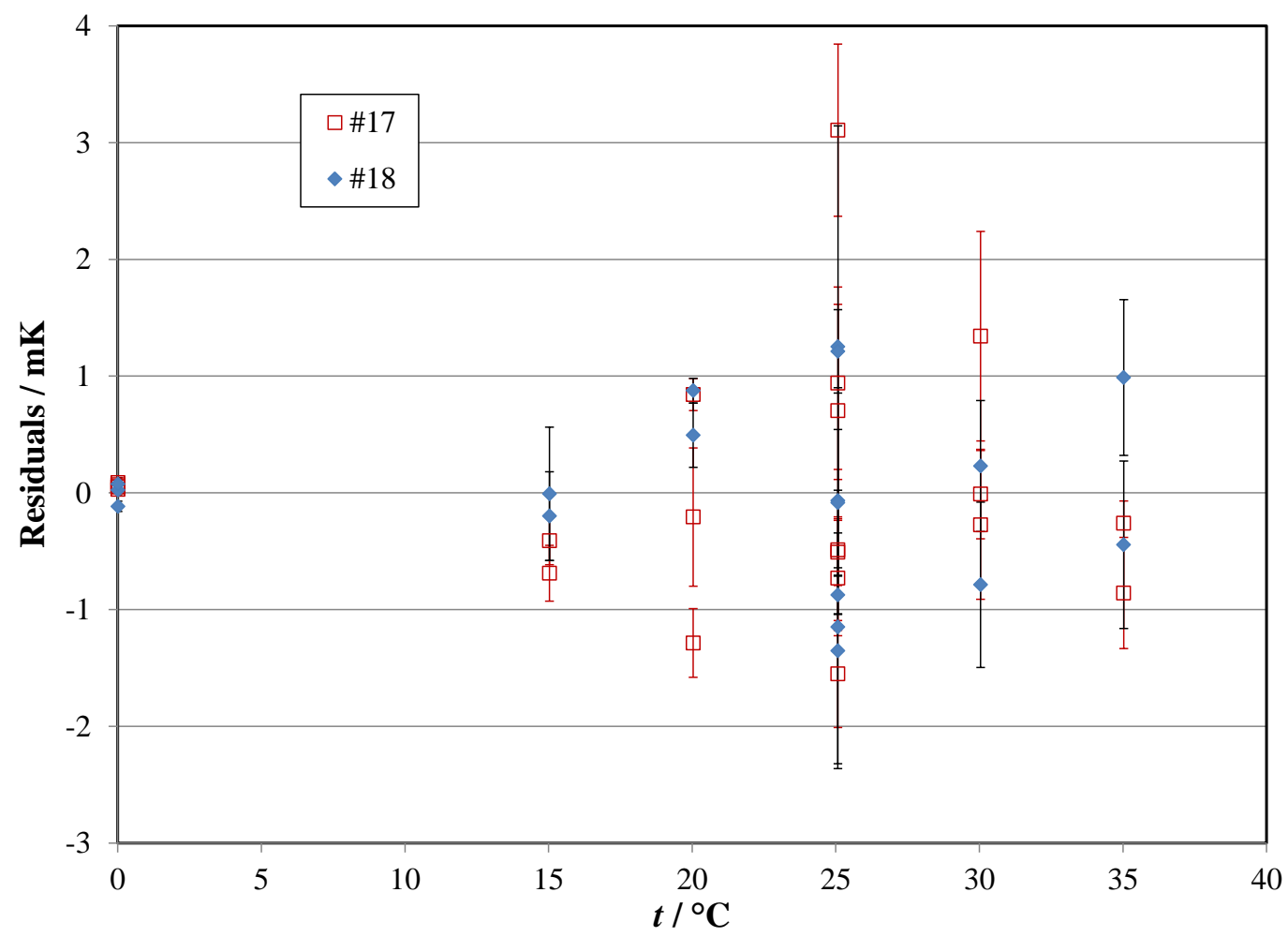

Figure 6b. Fit residuals to Eqn. 1 for the two WW base sensors, $m=17,18$ for $0 \mathrm{~mA}$ data. The error bars represent standard statistical (i.e. nominally random) uncertainty only. 


\subsection{Immersion Errors}

The primary sensor calibrations as described above are appropriate for conditions where heat transfer to the walls of the chamber is dominated by strong gas-phase diffusion or convection. The primary sensor $(n=13,14)$ calibrations are based exclusively on the zero-power, heliummediated resistance data, (i.e. $R_{n_{-} \mathrm{Cal}}(t)$ is the Eqn. 1 fit to $R_{n_{-} \mathrm{He}}(t, P=0)$ ). It is assumed that immersion errors under these conditions are negligible (see Discussion, section 4.1.1). Under vacuum conditions, however, immersion errors may be significant if there are departures from isothermal conditions. The procedures required for calibration of the sensors using the test chamber necessitated imposing significant departures from isothermal conditions as indicated by the base sensor temperatures. Hence, immersion errors were observed in the vacuum data during the calibration of the probes in the test chamber, and may also be present when the probes are installed in the $\mathrm{Sr}$ II chamber.

The immersion errors $R_{n_{-} \mathrm{Vac}}-R_{n_{-} \mathrm{He}}$ were found to be proportional to the differences, $t_{m}-t_{\mathrm{b}}$ ath , between a given base temperature $t_{m}$, as indicated by the base sensors $(m=15,16)$, and the bath temperature $t_{\text {bath }}$. The related errors $R_{n_{-}} \mathrm{Vac}-R_{n_{-} \mathrm{Ar}}$ were found to exhibit similar dependencies. While not actually a gradient, we use the term 'axial gradient' as a substitute term for the differences, $t_{m}-t_{\text {bath }}$, since any such gradients in the probes will be proportional to that difference. This representation of the data produces the immersion error curves shown in Figure 7 as shown with quadratic fits in $t_{m}-t_{\text {bath. }}$.

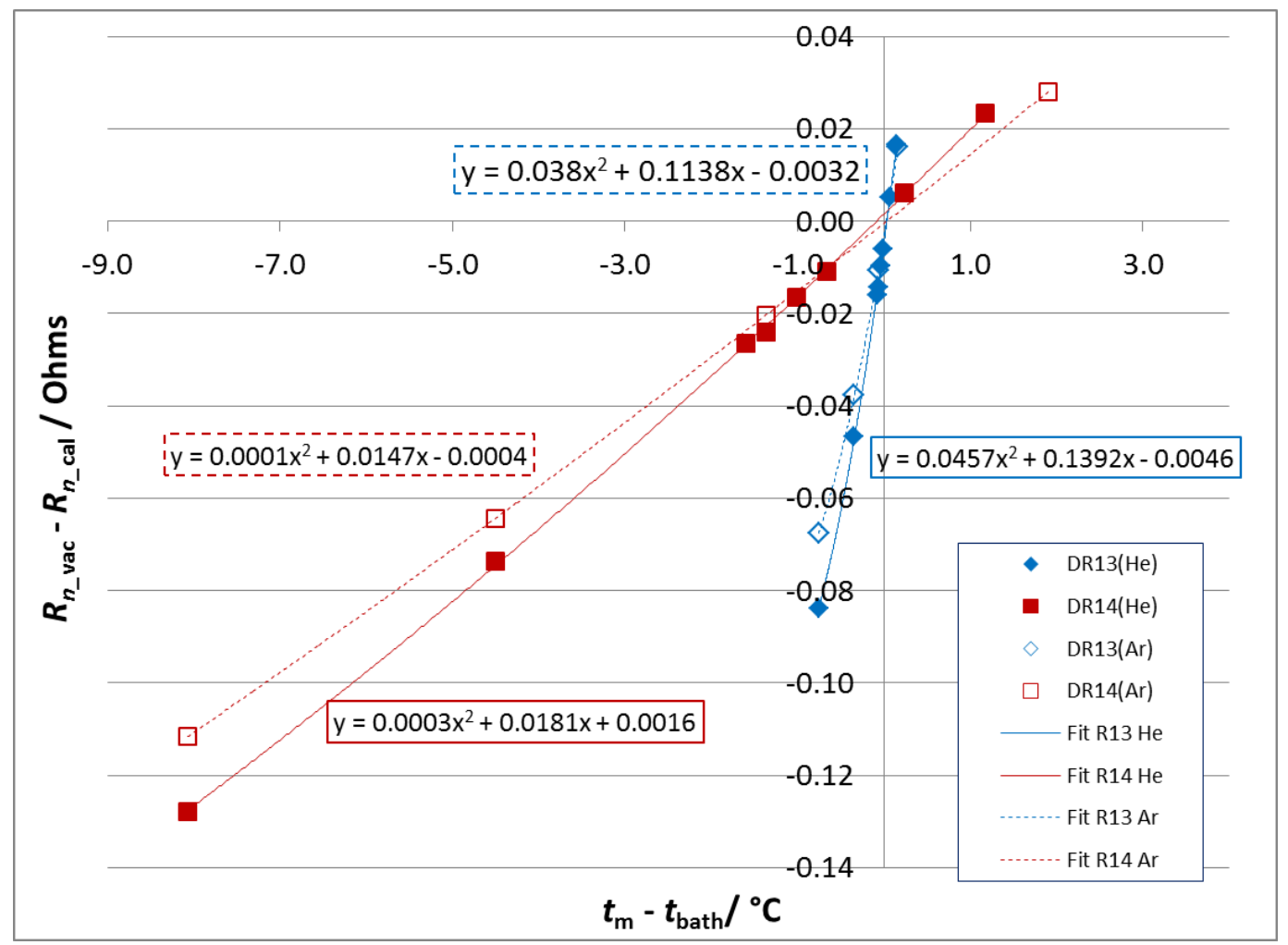

Figure 7. The complete set of immersion errors in Ohms versus the axial gradients $t_{m}-t_{\text {bath }}$ for both $\mathrm{He}$ and Ar data.

The quadratic fits shown in Figure 7 are unconstrained and unweighted. In principle the intercepts $\Delta_{n}$ should be zero, but the data are best fit with small offsets of a few $\mathrm{m} \Omega$, equivalent 
to $5 \mathrm{mK}$ to $10 \mathrm{mK}$. The quadratic term is only necessary to accommodate the largest values for the axial gradients. By limiting the range of data to a range the equivalent of $\pm 0.13{ }^{\circ} \mathrm{C}$ in $R_{n_{-} \mathrm{Vac}}-$ $R_{n_{-} \mathrm{He}}$ and to $\pm 1.8^{\circ} \mathrm{C}$ in $t_{m}-t_{\text {bath }}$, more linear regions of the error curves are observed, as shown in Figure 8. Table 8 is a compilation of the linear immersion error parameters derived from the He calibration data as shown in Figure 8.

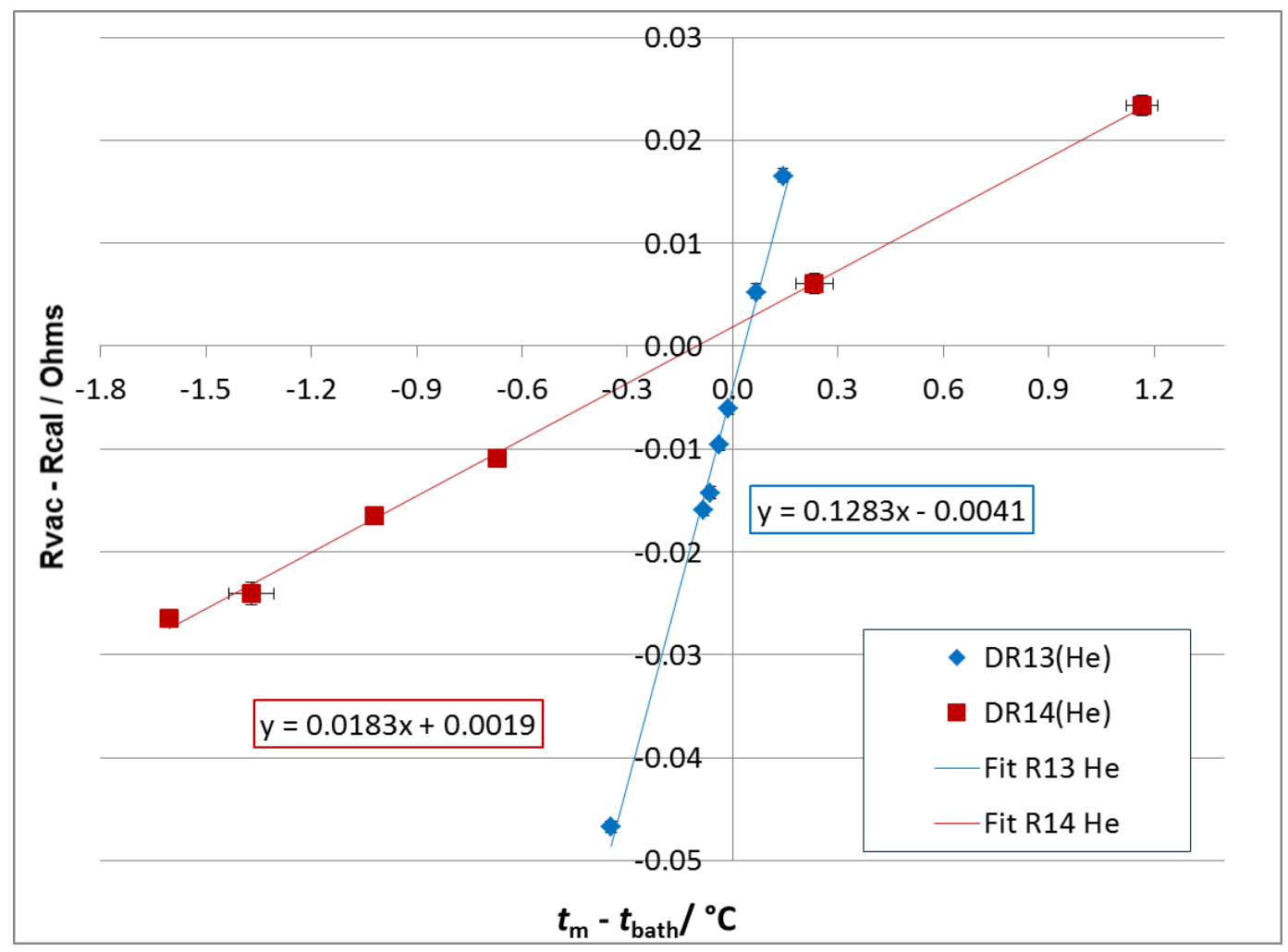

Figure 8. Linear portions of the Immersion Error curves for the vacuum and helium data.

Table 8. Least-squares fitting estimates for the linear immersion error coefficients for the two probes. The $C_{m n}$ are the immersion error coefficients and $\Delta_{n}$ are ad hoc offset parameters.

\begin{tabular}{|c|c|c|c|c|}
\hline parameter & $m, n=13,15$ Value & $m, n=13,15$ Std. Error & $m, n=14,16$ Value & $m, n=14,16$ Std. Error \\
\hline$C_{m n} / \Omega \cdot \mathrm{K}^{-1}$ & 0.128 & 0.005 & 0.0183 & 0.0003 \\
\hline$\Delta_{n} / \Omega$ & -0.0041 & 0.0007 & 0.00187 & 0.00035 \\
\hline
\end{tabular}

The parameter values shown in Table 8 are limited to this particular range of $t_{m}-t_{\text {bath }}$ and a simple unconstrained linear model. Depending on the exact fitting model and range of data chosen, the immersion error coefficients $C_{n m}$ exhibit a range of ratios $7 \leq C_{13,15} / C_{14,16} \leq 8$, with most of that variability being due to probe $1(n=13, m=15)$. This degree of ambiguity is a more realistic indicator of the relative uncertainties in the $C_{n m}$ than the simple statistical estimates shown in Table 8 . The physical origin of the large ratio in coefficients is treated below under the Discussion section 4.1.5. 


\subsection{Use of the Base Sensors for Correcting Immersion Errors}

Under high vacuum conditions, corrections should be applied to account for offsets in the primary sensor temperatures from axial gradients and the corresponding immersion errors as shown in Figure 8. The base sensors are used to affect this correction via measurements of $t_{m}$ but where the difference $t_{m}-t_{\mathrm{n}}$ is not directly measured. The immersion errors are most readily parameterized in terms of the difference between the vacuum and helium-mediated resistance readings of the primary sensors, $R_{n_{-}}$Vac $-R_{n_{-} \text {Cal }}$. Each of the two base sensors is matched to the two probes as shown in Table 1. Base sensor 15 is installed on probe 1 to provide corrections for primary sensor 13, and base sensor 16 is installed on probe 2 to provide corrections for primary sensor 14. Other configurations may be used on the Sr II chamber with the WW base sensors.

In the $\mathrm{Sr}$ II chamber, the primary sensors will measure temperatures $t_{\mathrm{n}}$ in vacuum conditions, so to apply a correction we impose the condition $t_{\mathrm{n}}=t_{\text {bath }}$ to the immersion error equation,

$$
R_{n \mathrm{Vac}}-R_{n \mathrm{Cal}}\left(t_{n}\right)=C_{n m}\left(t_{m}-t_{n}\right)+\Delta_{n}
$$

where $C_{n m}$ are the immersion error coefficients and $\Delta_{n}$ are ad hoc offset parameters to account for scale offsets or other unknown errors, which should in principle be zero. By combining equations 1 and 2 and solving for $t_{n}$ we obtain,

$$
t_{n}=\frac{C_{n m}-R_{0 n} A_{n}}{2 R_{0 n} B_{n}}+\frac{\sqrt{\left(C_{n m}-R_{0 n} A_{n}\right)^{2}+4 R_{0 n} B_{n}\left(R_{n V a c}-R_{0 n}-C_{n m} t_{m}-\Delta_{n}\right)}}{2 R_{0 n} B_{n}},
$$

for the temperature $t_{n}$, in ${ }^{\circ} \mathrm{C}$, for either probe as a function of the vacuum resistance reading

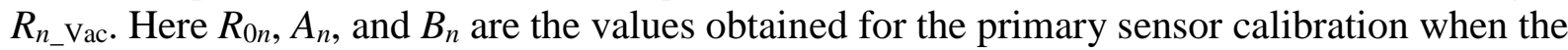
test chamber was under He exchange gas. The first term in Eqn. 3 is a positive constant for each probe $\left(2700{ }^{\circ} \mathrm{C}\right.$ and $3800{ }^{\circ} \mathrm{C}$, respectively), and the second term is always negative for the range of calibrations shown here and contains the dependence on the variables $R_{n_{-}}$Vac and $t_{m}$. In the limit in which $C_{n m}$ and $\Delta_{n}$ go to zero, Equation 3 reverts to the inverse of Eqn. 1.

For the purposes of applying corrections in the Sr II chamber, the immersion error coefficients must be considered model dependent and uncertain. Therefore, the recommended coefficients should not be derived from the statistical estimates from any one model (e.g. as in Table 8), but rather as a mean of an ensemble of different model estimates. In addition, the finite estimated values for $\Delta_{n}$ must be regarded as an indication of a deficiency in the data and or in the fitted model such that associated uncertainties should accommodate $\Delta_{n}=0$ within an assumed distribution of estimates. Thus we assume a relative uncertainty $u\left(\Delta_{n}\right) / \Delta_{n}=50 \%$. This treatment results in the recommended values and standard uncertainties for the immersion coefficients as given in Table 9. 
Table 9. Recommended values and standard uncertainties for the linear immersion error coefficients and offset parameters for the two probes.

\begin{tabular}{|c|c|c|c|c|}
\hline Parameter & $m, n=13,15$ Value & $m, n=13,15$ Std. Unc. & $m, n=14,16$ Value & $m, n=14,16$ Std. Unc. \\
\hline$C_{m n} / \Omega \cdot \mathrm{K}^{-1}$ & 0.1358 & 0.0075 & 0.0183 & 0.0006 \\
\hline$\Delta_{n} / \Omega$ & -0.004 & 0.002 & 0.002 & 0.001 \\
\hline
\end{tabular}

It should be noted that the Probe 1 immersion error coefficient is large by comparison to all other likely perturbations. When converted to an equivalent temperature change, the recommended $C_{13,15}=353 \mathrm{mK} / \mathrm{K}$ or $35.3 \%$. In contrast, and again from Table $9, C_{14,16}=47 \mathrm{mK} / \mathrm{K}$ or $4.7 \%$. The ratio of 7.5 can be seen as physically reasonable, despite the ratio of probe lengths of only 1.8 , given the exponential character of the immersion errors described in section 4.1.5 (Discussion). 


\section{Discussion and Uncertainties}

\subsection{Primary Sensors}

The most important assumptions being made for this calibration are that the test chamber was sufficiently isothermal during the data collection. However, the existence of axial gradients and immersion errors are due to known departures from isothermal conditions and must be quantified under known conditions. The two SPRTs were positioned on opposite sides of the test chamber in the water bath such that gradients from perturbation of the circulation pattern would produce nearly maximal differences. The SPRTs never disagreed by more than $2 \mathrm{mK}$ during the calibration and these differences have been factored into the uncertainty estimates for the $T_{\text {bath }}$ temperatures shown in Tables 2 and 4.

\subsubsection{Helium Data}

The low values $(<1 \mathrm{mK})$ for the residuals as shown in Figure 5 indicate that there were no sources of fluctuating errors or strongly non-linear systematic errors present with perturbations any larger than that uncertainty. The high degree of correlation in the $R(t)$ curves and residuals between the two probes also provides similar upper bounds on the degree to which immersion errors could have perturbed the helium calibration given the dissimilar probe lengths (see Immersion Effects below).

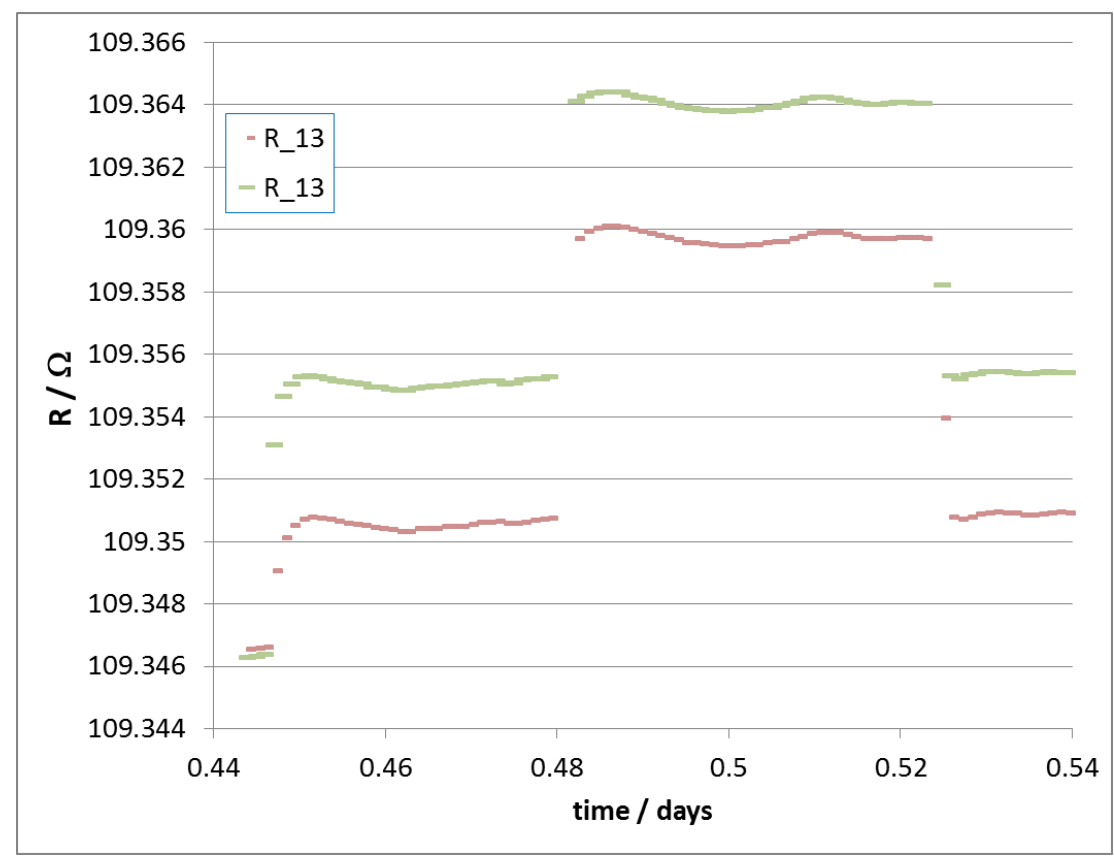

Figure 9. Primary sensor transient response to step changes in the selfheating power under helium exchange gas. Each point is $90 \mathrm{~s}=0.001 \mathrm{~d}$. Fluctuations are due to bath temperature instability of $\pm 1 \mathrm{mK}$.
The time constants for the primary sensors in helium were sufficiently fast that they were impractical to measure, with most of the transient response occurring within the $90 \mathrm{~s}$ time to scan all six measurement channels and thereby provide an update to any one particular channel. An estimated upper limit for the time constant is $\tau_{\text {He }} \lesssim 30$ s. A typical $50 \mu \mathrm{W}$ power change step response is shown in Figure 9. 


\subsubsection{Vacuum Data}

The vacuum data is used to calculate the error immersion coefficients only and a fit to Eqn. 1 is not necessary for that purpose. However, when such a fit is performed, the residuals are factors of 10 to 20 times those of the helium data fits as shown in Figure 5. These fits also fail to conform with the IEC specifications except over a very narrow range of temperatures, yielding low values for the mean temperature coefficient of $\alpha_{n}=0.003725^{\circ} \mathrm{C}^{-1}$ and $0.003665{ }^{\circ} \mathrm{C}^{-1}$ respectively. This systematic departure is also due to the influence of immersion errors. Overall, the vacuum data is less reproducible due to fluctuations and drift in the room temperature perturbations, which lead to the $3 \%$ to $5 \%$ uncertainties in the immersion coefficients.

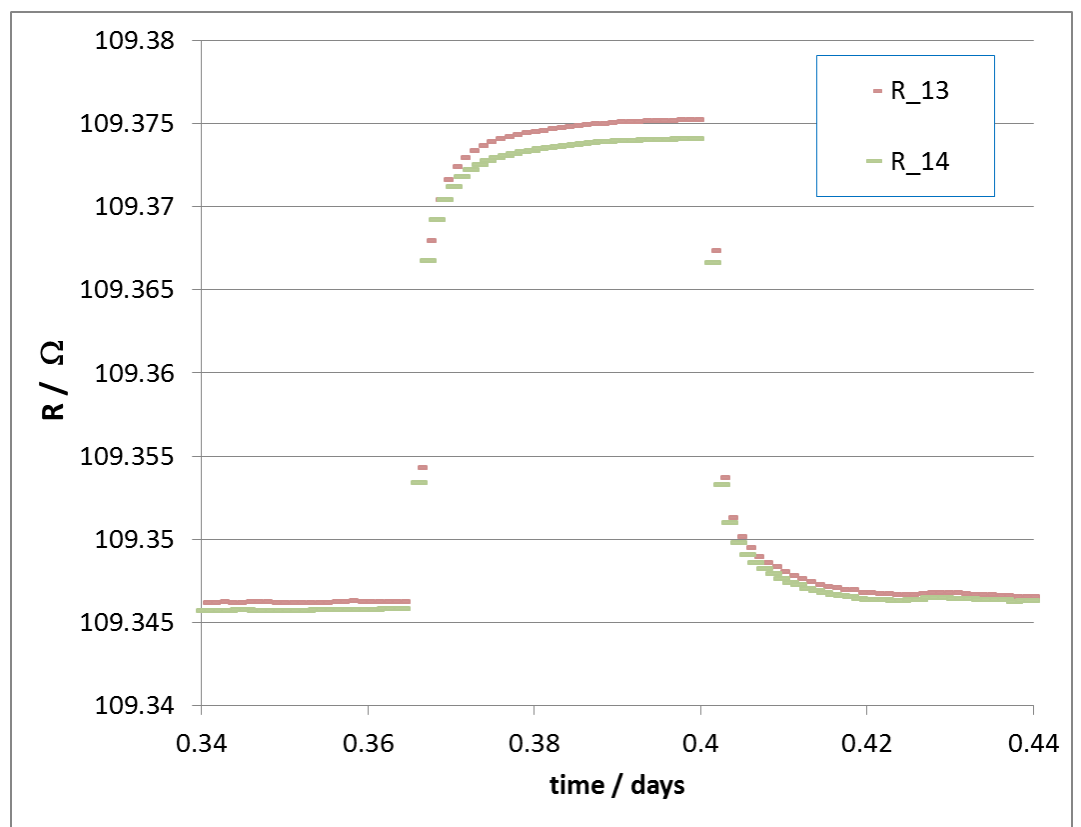

The observed time constants of the primary sensors under vacuum were $270 \mathrm{~s}$. The transient response to step changes of $50 \mu \mathrm{W}$ in the selfheating power are shown on Figure 10.

Figure 10. Primary sensor transient response to step changes in the self-heating power under vacuum. The increase of $0.5 \mathrm{~m} \Omega(1.3 \mathrm{mK})$ on the return to the base power level of $4 \mu \mathrm{W}$ is due to drift in room temperature during the elapsed $1.9 \mathrm{~h}$.

\subsubsection{Self-heating coefficients}

We expect that the slopes of the vacuum self-heating curves measured in the test chamber will reproduce in the Sr II chamber, but this should be checked at JILA after the sensors are installed. The best practice would be to measure the self-heating in-situ each time a temperature reading is obtained using the sensors with two or more currents and extrapolating to zero power. This practice will account for any differences which may exist in the self-heating coefficients between the test chamber and the Sr II chamber installations. Given this procedure, some increase in the random (i.e. type A) uncertainty will result in the $R(0)$ values, but no additional uncertainty will be incurred due to self-heating effects in the primary sensors.

A comparison of the observed self-heating coefficients is useful for understanding certain aspects of the heat transfer mechanisms. The self-heating coefficients of the primary sensors from 
Table 4 are shown in Figure 11 as a function of temperature. Some of the observed variability in $\eta_{\mathrm{He}}$ suggests that contamination of the helium with heavier impurities was a problem, particularly in the case of the $20{ }^{\circ} \mathrm{C}$ data. For this reason, these data were excluded from the average. The average values for the vacuum, argon, and helium self-heating coefficients are calculated from Table 4 and are equal to $1.5 \mathrm{mK} / \mu \mathrm{W}, 0.86 \mathrm{mK} / \mu \mathrm{W}$, and $0.45 \mathrm{mK} / \mu \mathrm{W}$, respectively. The ratios of coefficients are relatively small, only a factor of 1.9 separate the argon and helium coefficients and only a factor of 3.3 separate the vacuum and helium coefficients. In contrast, the thermal conductivities of helium and argon differ by a factor of 8.5. In addition, the time constant data suggest that the ratio of thermal resistances under vacuum conditions to helium conditions should be $>10$.

These observations can be reconciled by constructing a simple thermal resistance network with an internal resistance, $Z_{\text {int }}$, in series with an external resistance $Z_{\text {ext. }}$. The internal resistance is created by the fixed geometry and materials of the sensor package and is independent of the environmental conditions. In contrast, the external resistance is created by a combination of the variable and parallel thermal paths associated with the gas diffusion, $Z_{\mathrm{He}}$ or $Z_{\mathrm{Ar}}$, and radiation, $Z_{\mathrm{R}}$, heat exchange mechanisms.

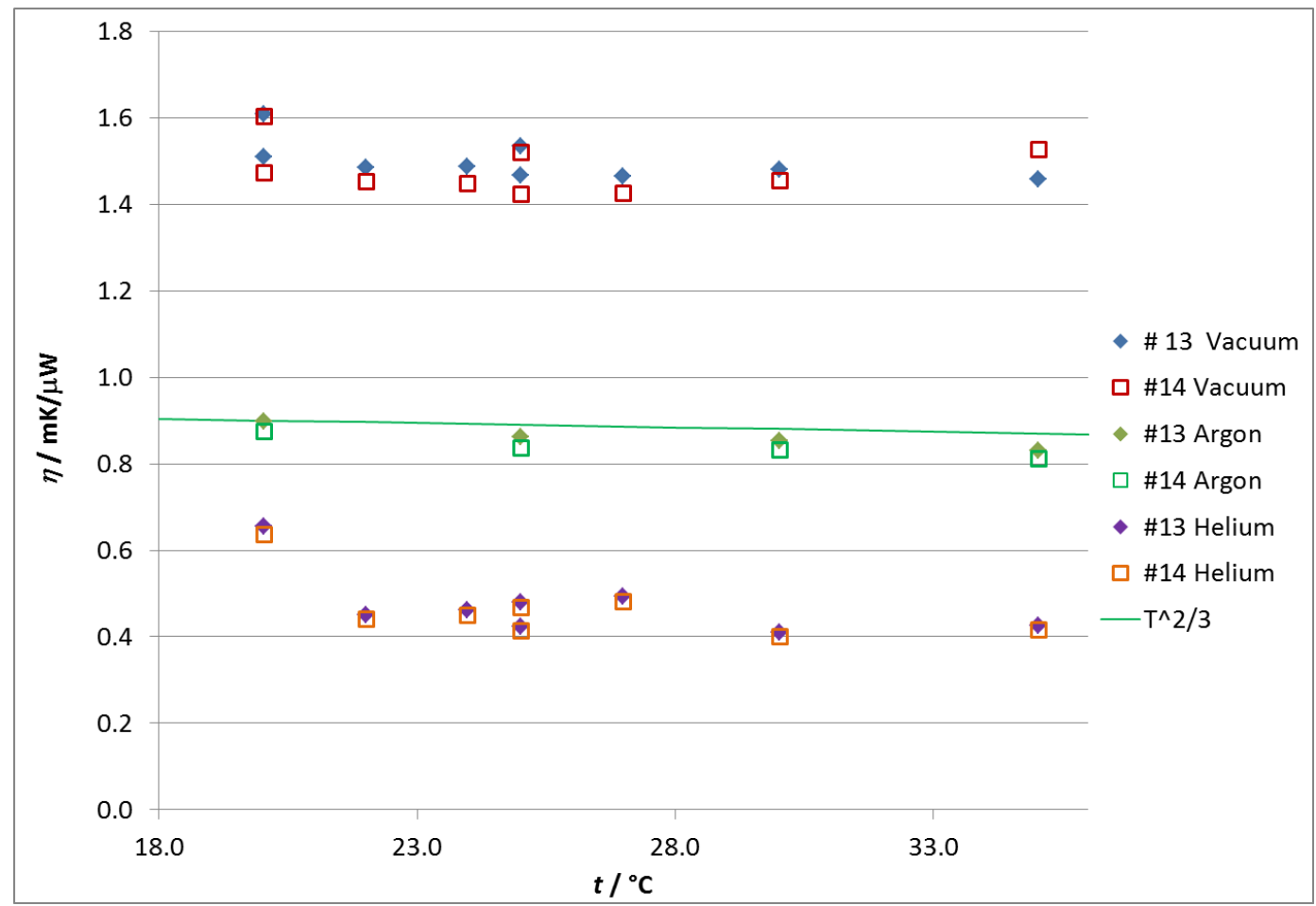

Figure 11. Self-heating coefficients of the primary sensors in helium, argon and vacuum.

Constructing three simple series/parallel equivalent circuits of the thermal resistances, using only $Z_{\mathrm{R}}$ and $Z_{\text {int }}$ under vacuum conditions and all three $\left(Z_{\mathrm{R}}, Z_{\text {int }}\right.$, and $Z_{\mathrm{He}}$ or $\left.Z_{\mathrm{Ar}}\right)$ under exchange-gas conditions, allows some estimates to be made of the values for these individual thermal resistances as follows,

$$
Z_{\mathrm{He}}=\frac{\left(\eta_{\mathrm{Vac}}-Z_{\mathrm{int}}\right)\left(\eta_{\mathrm{He}}-Z_{\mathrm{int}}\right)}{\eta_{\mathrm{Vac}}-\eta_{\mathrm{He}}} ; Z_{\mathrm{Ar}}=\frac{\left(\eta_{\mathrm{Vac}}-Z_{\mathrm{int}}\right)\left(\eta_{\mathrm{Ar}}-Z_{\mathrm{int}}\right)}{\eta_{\mathrm{Vac}}-\eta_{\mathrm{Ar}}} ; \text { and } Z_{\mathrm{R}}=\eta_{\mathrm{Vac}}-Z_{\mathrm{int}}
$$


This simple underdetermined model may uniquely determine all four thermal resistances if we introduce the additional constraint of $8.5 \times Z_{\mathrm{He}}=Z_{\mathrm{Ar}}$, assuming that both argon and heliummediated heat exchange conditions are dominated by diffusive conductivity. The manufacturer specifies the $\mathrm{C} 416$ self-heating at $0.4 \mathrm{mK} / \mu \mathrm{W}$ at $0^{\circ} \mathrm{C}$, and when $Z_{\text {int }}=0.35 \mathrm{mK} / \mu \mathrm{W}$ the assumed argon/helium ratio of 8.5 is achieved. Under these assumptions we then estimate the four thermal resistances: $Z_{\text {int }} \approx 0.35 \mathrm{mK} / \mu \mathrm{W} ; Z_{\mathrm{He}} \approx 0.108 \mathrm{mK} / \mu \mathrm{W} ; Z_{\mathrm{Ar}} \approx 0.92 \mathrm{mK} / \mu \mathrm{W}$; and $Z_{\mathrm{R}} \approx 1.15 \mathrm{mK} / \mu \mathrm{W}$.

It should also be noted that this model yields $Z_{\mathrm{R}} \approx 10 Z_{\mathrm{He}}$. In contrast, a crude estimate for $Z_{\mathrm{R}}$ is given by $\left(Z_{\mathrm{R}}\right)^{-1} \approx 4 \varepsilon \sigma T^{3} A_{\text {Reff }}$ which yields $Z_{\mathrm{R}} \approx 9 \mathrm{mK} / \mu \mathrm{W}$ assuming an effective radiative-coupling area $A_{\text {Reff }}$ equivalent to the geometric area $A_{\mathrm{Geom}} \approx 1.8 \times 10^{-5} \mathrm{~m}^{2}$ based on the dimensions of the $\mathrm{C} 416$ alone. This clearly yields a gross overestimate in $Z_{\mathrm{R}}$ since it would result in self-heating and time constants a factor of 8 larger than observed. The most likely explanation is that the radiative-coupling area $A_{\text {Reff }}$ is much larger than the physical area of the sensor itself due to the bonding and proximity to the glass, which augments (by a factor 8) the area of local infra-red absorption.

\subsubsection{Electrical Resistance}

The combined uncertainties in the resistance bridge measurements and resistance standard uncertainty are well below $1 \mu \Omega / \Omega$. The limiting uncertainty concerns that stability of the IMP resistance value $R_{0}$ for which we assign a standard uncertainty of $1 \mathrm{~m} \Omega$, or $2.6 \mathrm{mK}$. The value for $R_{0}$ as a fitting parameter is uncertain only at the level of $0.5 \mathrm{~m} \Omega$ as long as no attempt is made to use Eqn. 3 to interpolate temperature below the $20^{\circ} \mathrm{C}$ lower limit of the primary sensor data. Since the sensors can not be checked at the IMP to form a ratio (i.e. a local resistance scale independent of the Ohm), it is important for JILA to have a calibrated resistance standard available to establish the Ohm in the Sr II laboratory to better than $5 \mu \Omega / \Omega$. This will keep resistance scale errors at or below an equivalent of $1.3 \mathrm{mK}$.

\subsubsection{Immersion Effects}

The test chamber was designed to co-locate the probes at a fixed immersion. Consequently, it was not practical to generate variable immersion data which would be the normal approach in the calibration of relatively short probes. In the absence of such data, we must rely on analytical models to help estimate uncertainties and check if the relative errors between the two probes are physically reasonable. The problem of immersion errors in practical thermometry has long been recognized, with various authors providing analytical treatments [12,13]. More recently, White and Jongenelen (W\&J) [14] have provided a solution based on the heat diffusion equation in one dimension. Some general aspects of the $\mathrm{W} \& \mathrm{~J}$ treatment are applicable under vacuum conditions if the departures from isothermal conditions are small. These solutions have the general form

$$
T(x)=T_{0}+\Delta T\left[K_{1} e^{(x-L) / \delta}+K_{2} e^{-(x-L) / \delta}\right]
$$

for a distribution of temperatures along the axis of a cylindrical probe with coordinate $x$, sensing temperature at an immersion $x=L$ relative to an isothermal zone transition at $x=0$ with $T_{0}, \Delta T, K_{1}$ and $K_{2}$ determined by the appropriate boundary conditions [15] (see Figure 12). The characteristic length scale is $\delta=\sqrt{k w / h}$, where $k$ is the bulk thermal conductivity of a tubular 
probe with wall thickness $w$ and transverse surface heat transfer coefficient $h$. If the probe has a total length $L_{i}>L$, and we identify $T_{0}=T_{\text {bath }}$, then it is straightforward to show that,

$$
\frac{C_{m n}}{R_{n 0} \alpha_{n}}=\frac{T_{n}-T_{b a t h}}{T_{m}-T_{b a t h}} \square\left(\frac{K_{1}}{K_{2}}+1\right) e^{-L_{i} / \delta}
$$

where $R_{0 n} \alpha_{n} \cong 0.385 \Omega \cdot \mathrm{K}^{-1}$ for all Pt100 sensors. In this approximation, the constants $K_{1}$ and $K_{2}$ are functions of surface heat transfer coefficients $h, k, w$, and $L$, (note that the boundary conditions involving $h$ as used by W\&J do not apply under vacuum). Assuming that the ratio $K_{1} / K_{2}$ is independent of $L$, the $C_{m n}$ coefficients will depend only on the total lengths $L_{i}$ of the probes and not on the actual immersion depth $L$. This result then implies that the immersion error curves observed in the test chamber will be equivalent to those for the JILA Sr II chamber, despite the fact that the effective immersion depth $L$ will likely be different. This important result follows from the fact that we have an implicit boundary condition of $t_{m}$ at $x=L-L_{i}$. We also expect that the characteristic length $\delta$ and the constants $K$ should all be the same for the two probes since they are of identical construction, and only differ in their total length $L_{i}$.

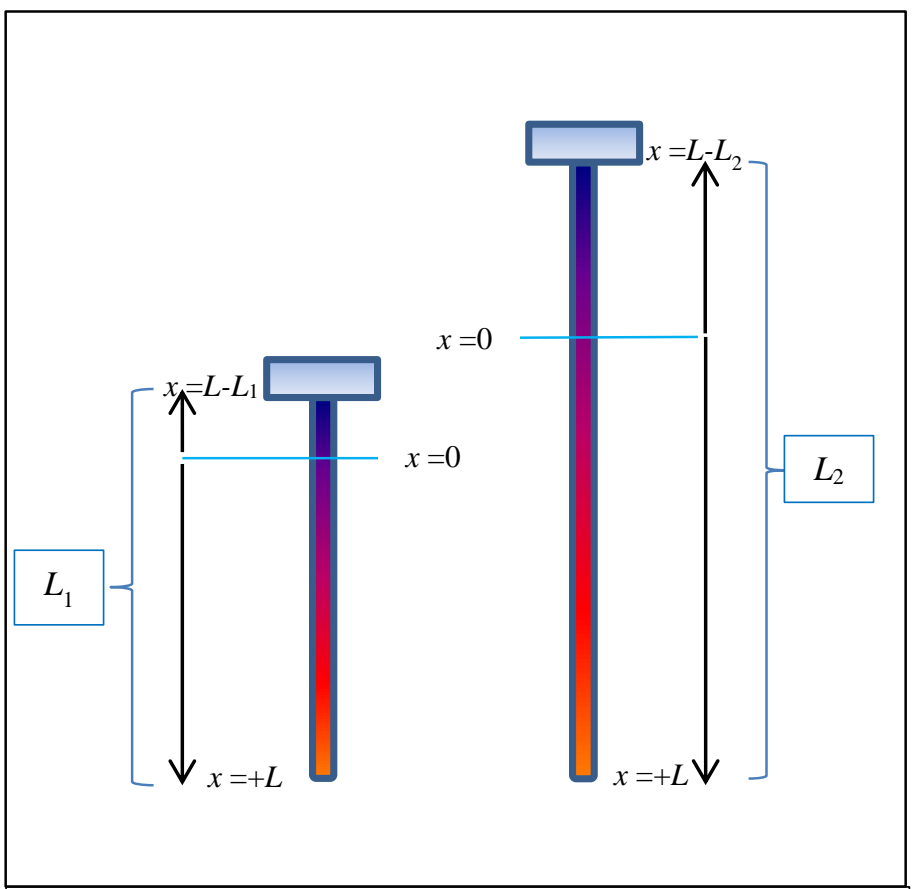

Figure 12. Axial coordinates for the probe immersions. Note the coordinate $x$ is negative for upward displacements. The difference in the effective immersion lengths $L$ for the two probes is exaggerated, but deliberate (see text).
The exponential factor in Eqn. 6 is the reason the immersion error coefficients are so different (e.g. $\times 7.5)$ for the two probes despite their lengths differing by less than a factor on 2. Assuming the characteristic length $\delta$ is identical for both probes, it may be estimated by taking the ratio of the $C_{m n}$ coefficients for the two probes via Eqn. 6. Taking values from Table 1 and Table 8 results in the estimate, $\delta=\left(L_{2}-L_{1}\right) / \ln (7.5) \cong 31 \mathrm{~mm}$ in vacuum.

One complicating issue concerns the effective immersion for probe 1 as installed in the test chamber. In this case a water-tight shroud was installed on the chamber to allow the base flange of the probe to be located under the water line but remain dry as shown in Figure $1 \mathrm{~b}$. Despite being submerged below the water line, the air pocket created by the shroud allowed the base flange to remain at higher (or lower) temperatures

than the bath, creating an effective immersion length $L$ that was different (i.e. shorter, as shown in Figure 12) from the geometric immersion length. The effective total length, however, should remain unaltered from the geometric total length $L_{1}$. 
Axial gradients are always present in any normal immersion-type thermometer probe. These gradients may or may not create significant immersion errors depending on the relative strength of the axial heat transfer (e.g. $k$ in this case) to that of the transverse heat transfer (e.g. $h$ ). The competition between these two heat transfer mechanisms/paths is what determines the characteristic length $\delta=\sqrt{k w / h}$. An implicit assumption in this calibration is that $\delta_{\mathrm{He}}<L_{n}$ and more specifically that $\left(K_{1} / K_{2}+1\right) e^{-L_{n} / \delta_{\mathrm{He}}} \leq \approx 10^{-4}$ in helium. Given the empirical estimate for $\delta_{\mathrm{Vac}} \cong 31 \mathrm{~mm}$ above, we can further estimate $\delta_{\mathrm{He}} \cong\left(h_{\mathrm{Vac}} / h_{\mathrm{He}}\right)^{1 / 2} \delta_{\mathrm{Vac}}$. Assuming that $h_{\mathrm{Vac}}$ is entirely radiative, and that $h_{\mathrm{He}}$ is entirely due to kinetic gas-diffusion conductance, a crude estimate for the ratio is given by [16],

$$
\frac{h_{\mathrm{Vac}}}{h_{\mathrm{He}}} \approx \frac{4 \sigma T^{3}}{\left(\frac{1}{\varepsilon_{a}}+\frac{1-\varepsilon_{b}}{\varepsilon_{b}}\left(\frac{a}{b}\right)\right)} \frac{a \ln (b / a)}{\lambda_{\mathrm{He}}}
$$

for concentric cylinders of radii $a \sim 3.5 \mathrm{~mm}$ and $b \sim 11 \mathrm{~mm}$ of emissivities $\varepsilon_{a} \sim 0.95$ and $\varepsilon_{b} \sim 0.25$ and where $\sigma$ is the Stefan-Boltzmann constant and $\lambda_{\mathrm{He}} \cong 0.16 \mathrm{~W} / \mathrm{m}-\mathrm{K}$ is the thermal conductivity of helium gas at $T=300 \mathrm{~K}$. Evaluating Eqn. 7 yields $h_{\mathrm{Vad}} / h_{\mathrm{He}} \cong 0.077$, which in turn implies that $\delta_{\mathrm{He}} \cong 8.6 \mathrm{~mm}$. This estimated value for $\delta_{\mathrm{He}}$ then allows estimations of the immersion attenuation factors $e^{-L_{i} / \delta_{\mathrm{He}}}$ to be calculated for helium yielding $2.3 \times 10^{-4}$ and $1.5 \times 10^{-7}$ for $i=1,2$ respectively. Thus, assuming that the currently unknown factor of $K_{1} / K_{2}$ for helium ${ }^{2}$ is $\lesssim 1$, it seems unlikely that there would have been measureable $(\$ 1 \mathrm{mK})$ immersion errors for either probe under conditions maintained during the helium exchange-gas calibration. This is consistent with the observed close correspondence between the helium fits for the two probes and correspondence of the fit residuals with bounds set by the SPRT data.

On the one hand, Eqn. 7 is only remotely applicable in the top few inches of the test chamber over which the $12.7 \mathrm{~mm}$ OD SS tubing surrounds the probes. Below that point, the chamber becomes more of a cavity and Eqn. 6 likely would under estimate $h_{\mathrm{Vac}}$ and the heat transfer ratio. On the other hand, if convection is possible in the helium, $h_{\mathrm{He}}$ would be underestimated and the ratio overestimated. Without any empirical or vetted theoretical information on the $K_{1} / K_{2}$ values in both helium and vacuum, it is not possible to make any further quantitative evaluations concerning the immersion errors.

The observed finite values for the $\Delta_{n}$ parameters in the immersion error curves indicates the presence of one or more systematic errors in the calibration data and or an inadequate immersion error model. Such deficiencies could be due to finite cross-immersion-error coefficients $C_{13,16}$ and $C_{14,15}$. We have analyzed the data to calculate only the diagonal coefficients $C_{13,15}$ and $C_{14,16}$. The existence of finite cross-coefficients $C_{13,16}$ and $C_{14,15}$ is also possible, but given the correlations in the base temperatures, it is not possible to provide unambiguous estimates for these parameters. In principle these are second order corrections (e.g. $C_{13,16} \propto C_{14,16} C_{13,14}$ ). We therefore assume these terms are negligible. The relatively large uncertainties assigned to the $\Delta_{n}$ parameters are partly an allowance for the possibility that this assumption is invalid.

\footnotetext{
${ }^{2}$ In the case of convective boundary conditions, the W\&J analysis suggests that $K_{1}<0$ and $K_{1} / K_{2}>-1$.
} 


\subsection{Base Sensors}

The base sensors serve two separate roles in this work. First, they served to provide the basis for determining the $C_{m n}$ immersion coefficients. That required an ex-post facto calibration using the same excitation current $(1 \mathrm{~mA})$ that was used during the test chamber measurements for calibration of the primary sensors. Secondly, the calibrated base sensors will allow monitoring of the temperatures $t_{m}$ as installed in the JILA Sr II chamber. In this case, there is a choice regarding the excitation current, including a zero extrapolation, since the calibration data included both $0.5 \mathrm{~mA}$ and $1.0 \mathrm{~mA}$ currents.

In the former case, there is an implicit self-heating offset included in the calibration, and this may not exactly match the thermal resistance as installed in the test chamber, increasing the calibration uncertainty. The self-heating coefficients observed during the base sensor calibrations were unusually low, only $0.18(4) \mathrm{mK} / \mu \mathrm{W}$, or approximately $19 \mathrm{mK}$ at $1 \mathrm{~mA}$ excitation. Despite this relatively low self heating, this introduced $4.4 \mathrm{mK}$ uncertainty component into the $1 \mathrm{~mA}$ data used for the determination of $t_{m}$ and $C_{n m}$.

For the purposes of monitoring the base temperatures in the Sr II chamber, a slightly lower overall uncertainty can be achieved by using the $0 \mathrm{~mA}$ coefficients and extrapolating two-current data in the same way as done for the primary sensors.

Once the preliminary uncertainty analysis was completed, it became evident that the less-thansatisfactory reproducibility of the TFPRT base sensors had contributed to the uncertainty in the immersion coefficients $C_{m n}$ and in particular the finite $\Delta_{n}$ parameters. While nothing more could be done to improve those uncertainties ex post facto, it was decided to replace the TFPRT base sensors for the purposes of future use at JILA on the Sr II chamber. The WW PRTs $m=17,18$ were calibrated for this purpose and achieved much lower calibration uncertainty as already noted in section 3.2.2. These WW base sensors should be used exclusively with $0 \mathrm{~mA}$ extrapolated resistance readings to achieve the lowest uncertainty on the Sr II chamber. 


\subsection{Pressure dependence under vacuum conditions}

There are two issues concerning residual pressure under nominal vacuum conditions that were investigated. The first is how the pressure inside the chamber as indicated by the Varian 24 ion gauge differed from the pumping system ion gauge which was used for all routine monitoring. This was necessary due to the restrictions on routine use of the Varian 24 gauge due to excessive chamber heating. The second issue is establishing the threshold pressure under which no further changes could be observed in the equilibrium (i.e. net heat transfer) for the primary sensors. This was necessary to establish when the vacuum was low enough to begin taking 'vacuum' data.

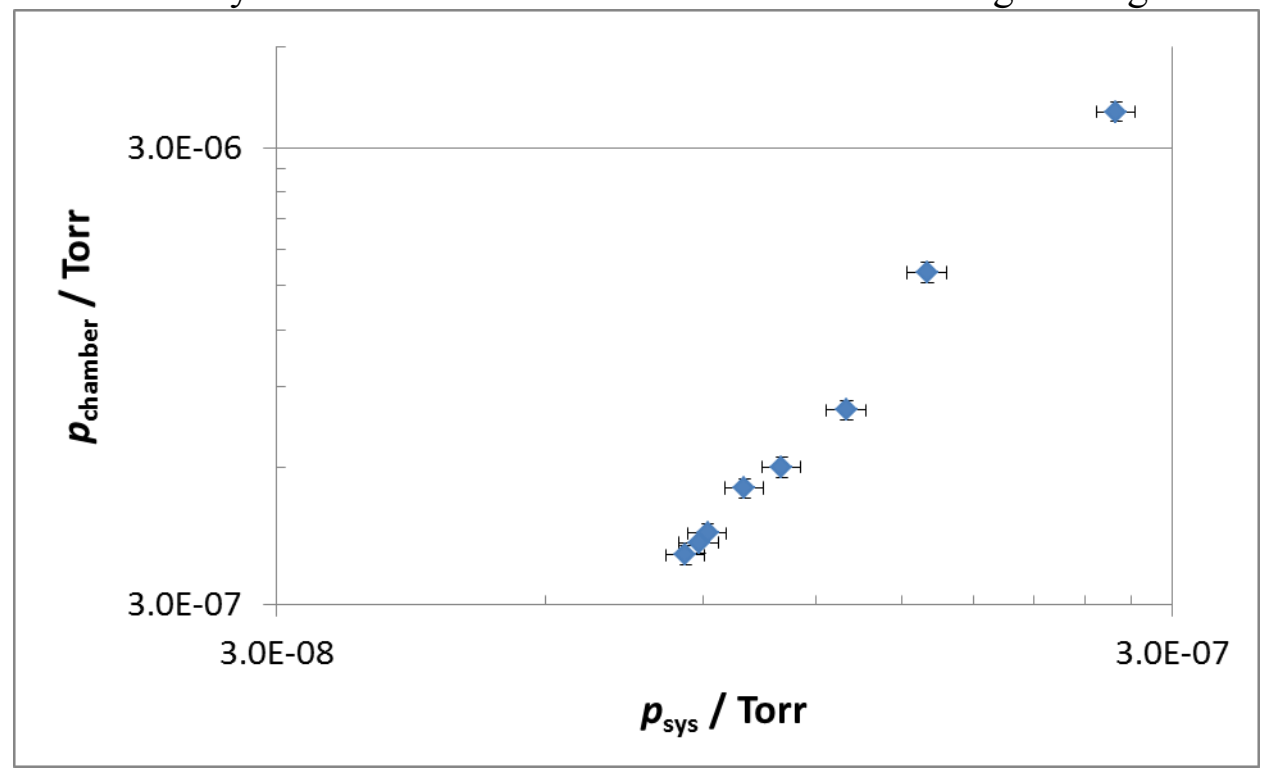

Figure 13. Relationship for the system pressure and internal test chamber pressure. Data are shown with nominal $5 \%$ standard uncertainties.

A test was conducted to compare the system pressure $p_{\text {sys }}$ with the test chamber pressure $p_{\text {chr }}$ using the two ion gauges as already described in the section 2.2.2 under 'Procedures'. The results are shown in Figure 13. For system pressures below $1 \times 10^{-7} \mathrm{Torr}\left(1.3 \times 10^{-5} \mathrm{~Pa}\right)$, the test chamber pressure was at or below $5.4 \times 10^{-7}$ Torr $\left(7.2 \times 10^{-5} \mathrm{~Pa}\right)$ as read on the Varian 24 ion gauge installed in the chamber.

Another test was conducted to monitor the primary sensors while pumping out the chamber from $\sim 2$ Torr $(270 \mathrm{~Pa})$ helium to a pressure just below $1 \times 10^{-7}$ Torr $\left(1.3 \times 10^{-5} \mathrm{~Pa}\right)$ (as read on the system ion gauge) at a constant bath temperature of $25.01{ }^{\circ} \mathrm{C}$. During this test the ambient laboratory temperature was $\approx 22.8{ }^{\circ} \mathrm{C}$ and the base temperatures were $t_{15} \cong 24.974(2)^{3}{ }^{\circ} \mathrm{C}$ and $t_{16} \cong 23.995(8){ }^{\circ} \mathrm{C}$. During this test the excitation currents were held constant at $200 \mu \mathrm{A}$ (i.e. $4.4 \mu \mathrm{W}$ ) and the bath temperature remained constant to within $\pm 1 \mathrm{mK}$. The results are shown in Figure 14. As the chamber is pumped out, the sensors cool down to a new equilibrium as determined by the immersion error under vacuum conditions. The offset for the \#14 sensor is because the difference $t_{16}-t_{\text {bath }} \approx-1{ }^{\circ} \mathrm{C}$ while in contrast $t_{15}-t_{\text {bath }}$ was approximately a factor of 27 smaller. Once the system pressure reached $1.0 \times 10^{-7}$ Torr $\left(1.3 \times 10^{-5} \mathrm{~Pa}\right)$, no further changes in the sensor's resistances could be resolved.

\footnotetext{
${ }^{3}$ Numbers in parentheses indicate the standard uncertainly in the least significant digit.
} 


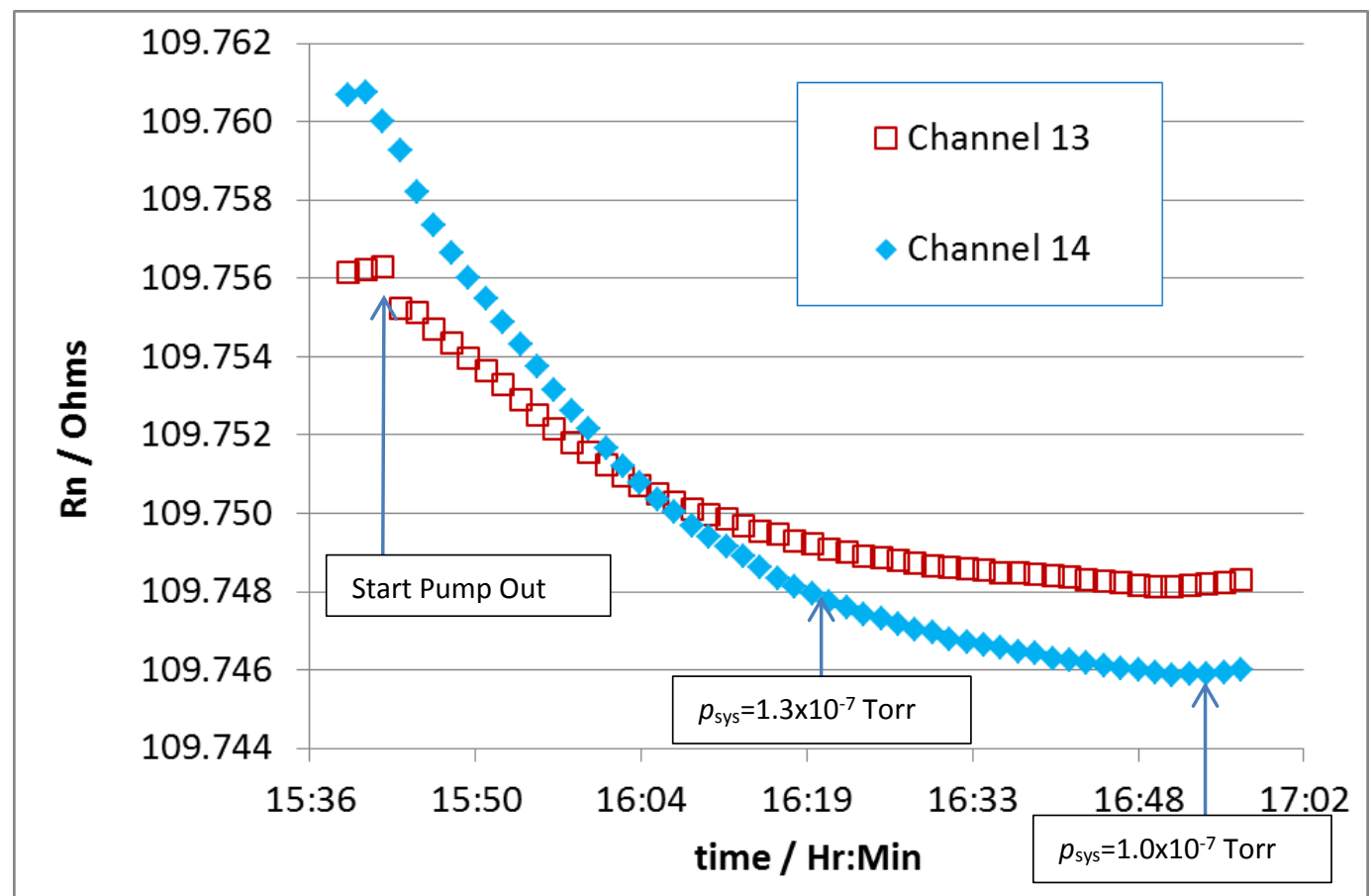

Figure 14. The equilibration of the primary sensors during a pump out from helium to vacuum.

These data suggest that once the system pressure reaches $\approx 10^{-7}$ Torr $\left(10^{-5} \mathrm{~Pa}\right)$ or less, the mean free path of the residual gases has become sufficiently large and the gas density so low that gasmediated heat exchange is negligible. At these pressures we should expect that BBR coupling is the dominant heat exchange mechanism. To the extent that there still remains some small (i.e. unresolved) influence from gas diffusion under these conditions, it would represent a negligible contribution compared to the statistical uncertainties already assigned to the immersion error coefficients $C_{n m}$.

\subsection{Uncertainty Curves}

The uncertainty in $t_{n}$ as determined by Eqn. 3 can be calculated given the values of the fixed parameters, realistic ranges of values for the variables $R_{n_{-} \text {Vac }}$ and $t_{m}$, and estimated nonparametric standard uncertainties as shown in Table 10. These are then combined with the parametric uncertainties given in Tables $5 \mathrm{~b}, 6$, and 8 . Examples of the estimated total standard calibration uncertainties as a function of the measured vacuum resistance $R_{n_{-}}$Vac are shown in Figures $15 \mathrm{a}$ and $15 \mathrm{~b}$ for each of the two probes in the case where $t_{m}=23{ }^{\circ} \mathrm{C}$.

Table 10. Summary of non-parametric standard uncertainties for the primary and base sensors.

\begin{tabular}{|r|c|c|}
\hline Source & $u_{n} / \mathrm{mK}$ & $u_{m} / \mathrm{mK}$ \\
\hline non-uniformity & 1 & 2 \\
\hline SPRT calibration & 1 & 1 \\
\hline temperature stability & 1 & 1 \\
\hline self-heating & 0.5 & 4 \\
\hline & & \\
\hline Resistance Measurement & 0.07 & 0.07 \\
\hline$u\left(t_{\text {cal }}\right)=\mathrm{RMS}$ & 1.8 & 4.7 \\
\hline
\end{tabular}




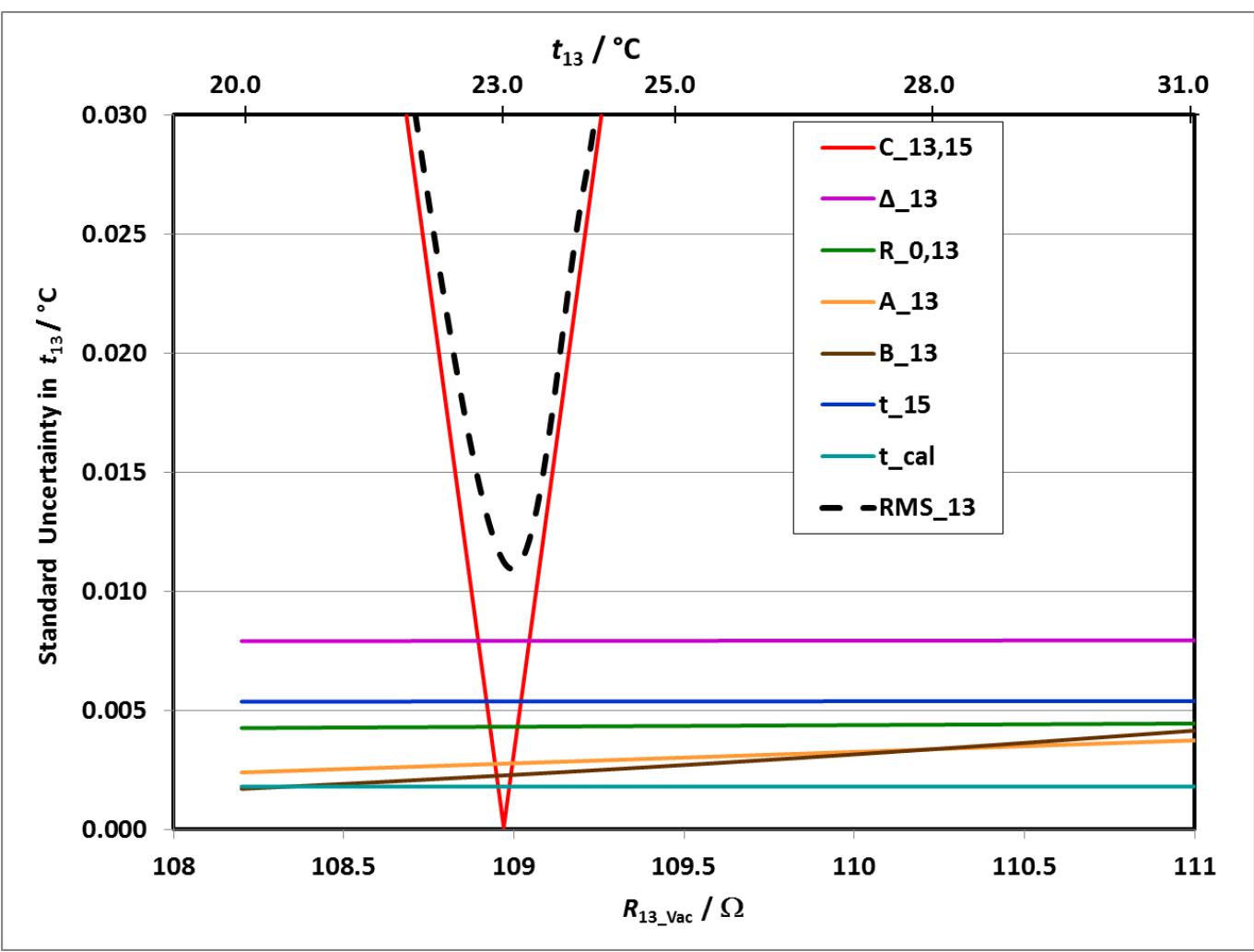

Figure 15a. Standard uncertainty components and combined total uncertainties in measured temperature $t_{13}$ when using probe 1 with the $n=13$ sensor to determine temperature in the Sr II vacuum chamber.

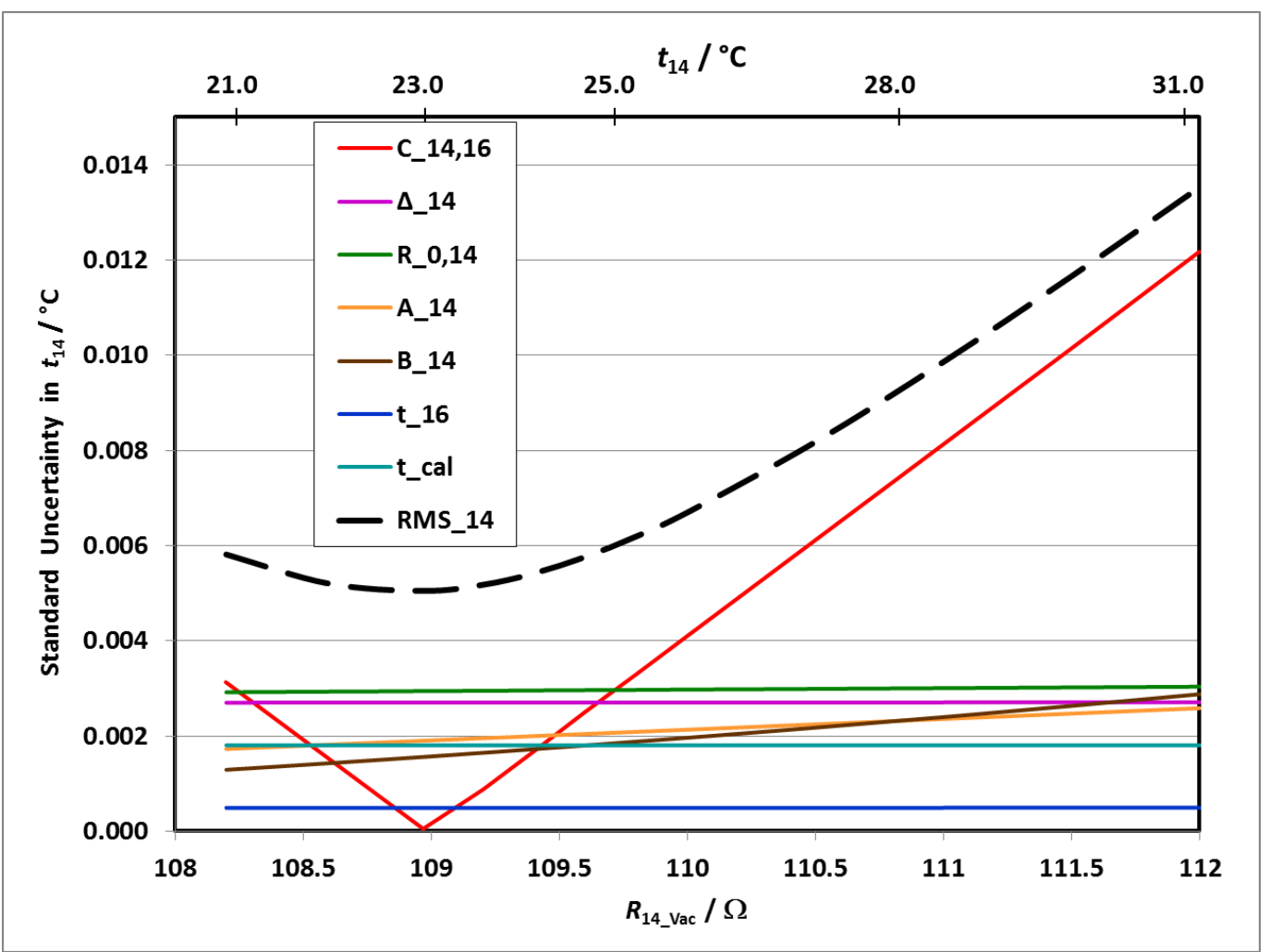

Figure 15b. Standard uncertainty components and combined total uncertainties in measured temperature $t_{14}$ when using probe 2 with the $n=14$ sensor to determine temperature in the Sr II vacuum chamber. 
From Figure 15a the strong influence of axial gradients and immersion errors in probe 1 is clearly evident. For this example $t_{\mathrm{m}}=23{ }^{\circ} \mathrm{C}$, and when $R_{\mathrm{n}_{-} \mathrm{Vac}} \approx 109 \Omega, t_{n}=23{ }^{\circ} \mathrm{C}$ and the axial gradients are null, resulting in a relative minima as the associated immersion errors go to zero. Departures from the uniform condition cause large uncertainties in probe $1 /$ sensor 13 , but these are much more gradual for the longer probe $2 /$ sensor 14 (Figure 15b) with a smaller immersion error coefficient. Similar curves may be generated when the value for $t_{\mathrm{m}}$ is varied.

\subsection{Deviation of $\boldsymbol{T}_{\mathbf{9}}$ from thermodynamic temperature}

All temperatures presented here are directly traceable to the NIST realizations of the ITS-90. The ITS-90 was designed to define temperatures $T_{90}$ as close approximations to thermodynamic temperature $T$ and consistent with the SI kelvin. The ITS-90 definitions [17] were based on the best available information on thermodynamic temperature prior to its adoption in 1990. Since that time, new and more accurate methods have become available which have allowed a more accurate assessment of $T-T_{90}$ [18]. The Consultative Committee on Thermometry (CCT) operating under the Committee International de Poids et Mesures (CIPM) have published recommended values for $T-T_{90}[19]$. In the limited range between $0{ }^{\circ} \mathrm{C}$ and $35^{\circ} \mathrm{C}$ considered here, the deviation is approximately quadratic in $t /{ }^{\circ} \mathrm{C}=T-273.15$, and given by $T-T_{90} / \mathrm{mK} \approx 0.127 t+6.13 \times 10^{-4} t^{2}$, or $\sim 3.9(4) \mathrm{mK}$ at $300 \mathrm{~K}(\sim 13 \mu \mathrm{K} / \mathrm{K})$. Given the current uncertainty requirements for the $\mathrm{Sr}$ II clock are approximately $10 \mathrm{mK}$, and that the BBR spectral power scales as $T^{4}$, this correction could be considered as an optional final step in determination of the $\mathrm{Sr}$ II BBR temperature, but is probably not critical. 


\section{Sr II Installation and Results}

\subsection{Shipping and installation}

After the sensors were returned to JILA, they were checked for agreement to ensure that no calibration shifts occurred in the shipping process. The sensors agreed within a few $\mathrm{mK}$, which is well within their combined total uncertainty of $12 \mathrm{mK}$. The magnitude and sign of TFPRT calibration shifts are not significantly correlated with time, vibration, or minor thermal excursions. Hence, even though the two sensors are of the same make and model and under the same nominal external stresses, the probability of these exhibiting identical calibration shifts is small. The sustained agreement between the two sensors after shipping gives us confidence that calibration shifts during transport were negligible.

The test chamber was baked at $100{ }^{\circ} \mathrm{C}$ and pumped down to $3 \times 10^{-10}$ Torr $\left(4 \times 10^{-8} \mathrm{~Pa}\right)$ to ensure that the sensors remained compatible with vacuum at that level. Baking the sensors was not expected to shift their calibrations appreciably since they were preselected for robustness against thermal cycling up to $200{ }^{\circ} \mathrm{C}$ (see section 1). The sensors and their glass mounts were then transferred from the test chamber to the Sr II vacuum chamber using a gas backflow. This procedure involves venting the Sr II chamber to high-purity nitrogen at a pressure of $\sim 20 \mathrm{kPa}$ greater than the atmospheric pressure in Boulder. Under these conditions, removing conflats from the Sr II chamber to make room for the sensors does not cause measurable contamination from the laboratory air due to the positive pressure of the nitrogen gas. The sensors were installed in the presence of this positive pressure, and then the chamber was pumped down. Cartridge heaters were used to locally bake the glass tubes at $150{ }^{\circ} \mathrm{C}$ to remove any water vapor accumulated on the structures. The chamber returned to its usual pressure after two days of pumping.

\subsection{Removing heat sources and sinks}

The clock transition frequency shift due to BBR is

$$
\Delta v_{B B R}=v_{\text {stat }}\left(\frac{T}{T_{0}}\right)^{4}+v_{\text {dyn }}\left(\frac{T}{T_{0}}\right)^{6}
$$

where $v_{\text {stat }}$ and $v_{\text {dyn }}$ are constants that describe the atomic response to BBR and $T_{0}=300 \mathrm{~K}$. This equation is used to subtract out the BBR shift from the measured clock transition frequency. $v_{\text {stat }}$ and $v_{\text {dyn }}$ are precisely known only for an ideal blackbody spectrum, so a significantly nonthermal heat environment will make it impossible to apply a precise correction. Therefore, it is critical to ensure that the thermal environment inside the Sr II vacuum chamber is sufficiently uniform. To do this, we surrounded the Sr II chamber with a heat shield for passive temperature stabilization. We used infra-red (IR) thermometers and thermistors to confirm that there were no unexpected heat sources around the Sr II vacuum chamber. Known heat sources were switched off and on to see if they had a measurable effect on the sensors.

We were able to remove all heat sources that affected the sensors. The chilled water used to cool our magneto-optical trap coils was the only heat sink that had an appreciable impact on the sensor measurements. Since the cooling from the chilled water over compensated for the heating from the coils, creating a net heat sink, it proved important to change the temperature of the 
cooling water such that the coil heating and water cooling combined kept the coils at room temperature.

\subsection{Measuring temperature gradients}

Previously we used simulations of the Sr II vacuum chamber to show that systematic bias due to thermal perturbations is negligible for temperature gradients across the chamber of less than $150 \mathrm{mK} / \mathrm{cm}$ [1]. Our measurements with thermistors and an IR thermometer put an upper bound on chamber gradients of about $50 \mathrm{mK} / \mathrm{cm}$. For a better measurement of gradients inside the chamber, we attached our long sensor to the chamber with an edge welded bellows so that the sensor could be translated. The difference between sensor measurements for two positions of the long sensor $(2.5 \mathrm{~cm}$ apart ) infers a gradient of $0.57 \mathrm{mK} / \mathrm{cm}$ with a statistical uncertainty of $12 \mathrm{nK} / \mathrm{cm}$, which is several orders of magnitude smaller than any value that would concern us.

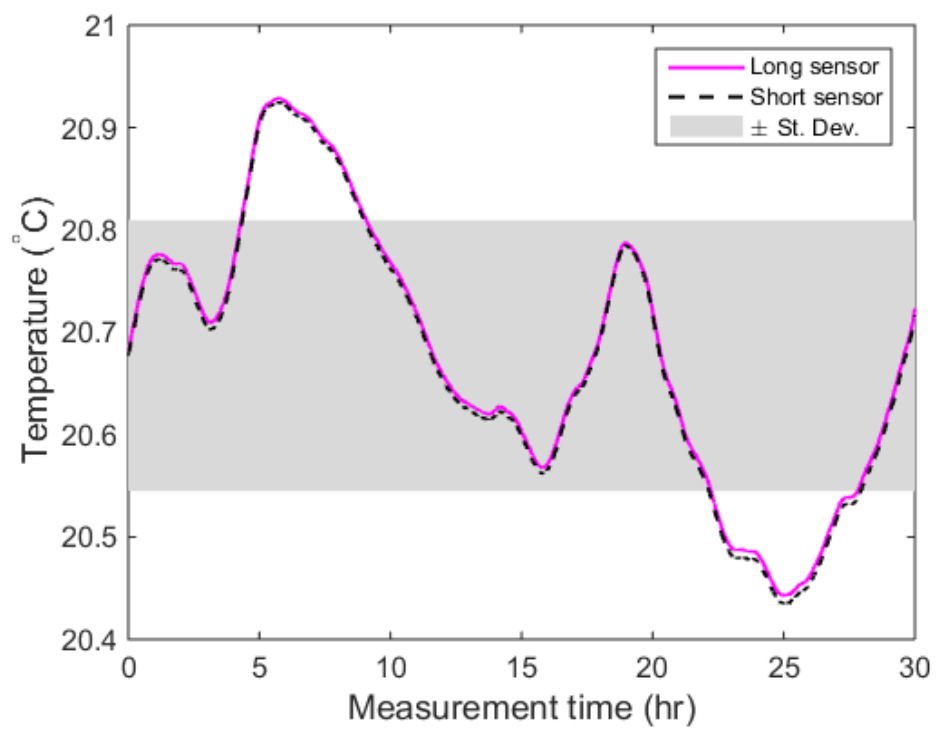

Figure 16. The sensors inside the Sr II chamber. Here heat sources and sinks have been removed and passive temperature stabilization is employed to make the temperature uniform inside the chamber. The standard deviation of the data (shown as a gray confidence band in the plot) indicates that the ambient temperature is stable to $\pm 130 \mathrm{mK}$

\subsection{Sensor agreement}

Running the Sr II apparatus in normal clock operation conditions, we observed agreement between the sensors of about $5 \mathrm{mK}$, which is within the $12 \mathrm{mK}$ combined uncertainty of both sensors. The base sensors did not show any measurable gradients across the sensor mounting structures. This confirms that calibration shifts due to sensor transport, installation, and thermal cycling are insignificant. Using the long sensor to infer the BBR shift, the $5 \mathrm{mK}$ standard uncertainty of this sensor yields a BBR shift relative uncertainty of $3 \times 10^{-19}$. This represents a factor of 5 improvement over our previous work. 


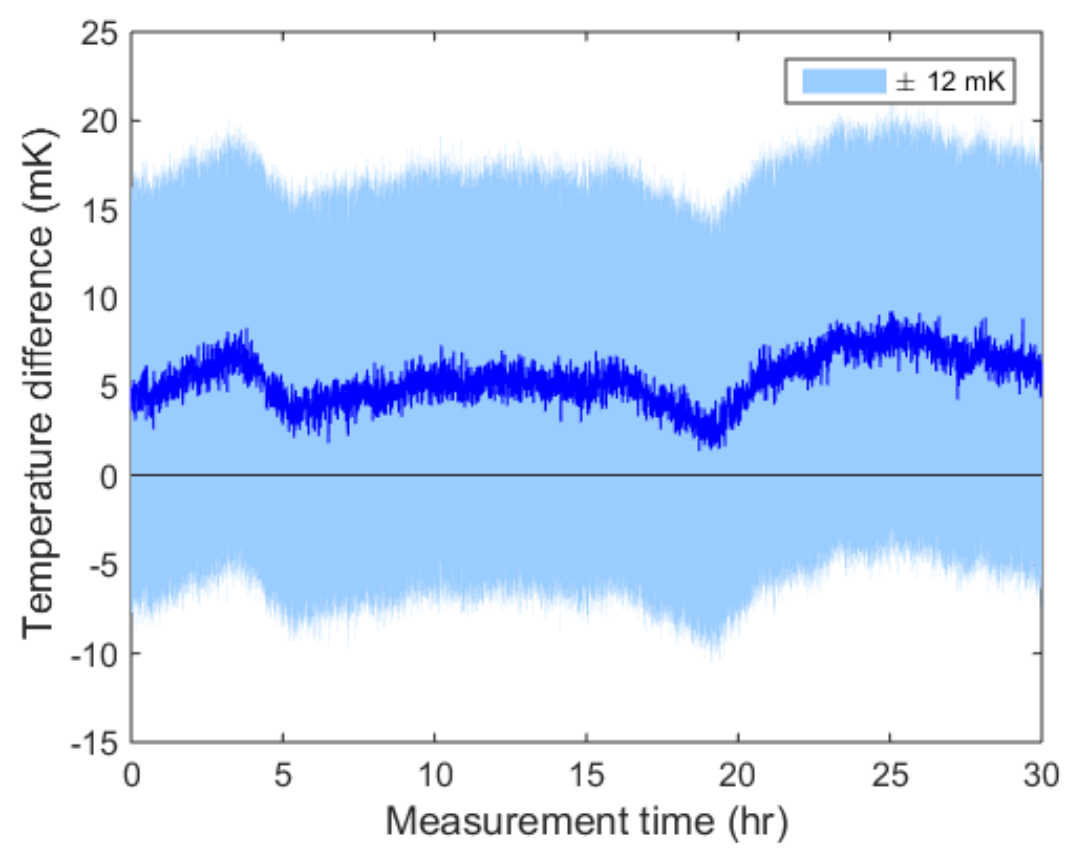

Figure 17. The difference between the long and short sensors. The measured difference between the sensors is within their combined standard uncertainty of $\pm 12 \mathrm{mK}$.

\section{Summary and Conclusions}

The two JILA Sr II temperature probes have been calibrated on the ITS- 90 over the range $20^{\circ} \mathrm{C}$ to $35^{\circ} \mathrm{C}$. The probes exhibit immersion errors under vacuum conditions which are functions of the measured mean axial gradient over the entire length of each probe. Each probe has a calibration for two sensors, primary and base, which together may be used to make in situ corrections of the immersion errors under vacuum. The relative errors are approximately 7.5 times greater for Probe 1 than Probe 2 for the same axial gradient. The achievable standard uncertainty for use of Probe 1 depends strongly on the departures from uniform (i.e. null gradient) conditions, with a sharp relative minimum of approximately $11 \mathrm{mK}$. Probe 2 exhibits a more shallow relative minimum in uncertainty of approximately $5 \mathrm{mK}$. This assumes that the combined resistance measurement uncertainties at JILA can be maintained at $5 \mu \Omega / \Omega$ or less and that the sensors are not exposed to temperatures exceeding $200^{\circ} \mathrm{C}$. Corrections for known differences between ITS-90 and thermodynamic temperature are considered as an option depending on the uncertainties actually achieved in practice. The sensors successfully retained their calibrations after being transferred to the Sr II vacuum chamber. Along with these accurate sensors, a well-engineered thermal environment inside the vacuum chamber enabled temperature measurements with standard uncertainties as low as $5 \mathrm{mK}$. 


\section{Acknowledgements}

The authors would like to thank Jim Fedchak of the NIST TMG for helpful advice and providing the use of an ion gauge and a controller. We also thank Howard Yoon of the NIST Sensor Science Division for helpful discussions and Dawn Cross and Wyatt Miller of the NIST TMG for assistance in the laboratory. We are grateful to Sara Campbell and George Marti for assistance with installing the sensors in the Sr II vacuum chamber and for laboratory support when the JILA-based sensor measurements were made. 


\section{References}

1. B. J. Bloom, T. L. Nicholson ,J. R. Williams, S. L. Campbell, M. Bishof, X. Zhang, W. Zhang, S. L. Bromley and J. Ye, Nature, 506, 71-75 (2014).

2. Heraeus Sensor Technology, Datasheet.

3. International Electrotechnical Commission, IEC 60751-2008, Platinum Resistance Thermometers and Platinum Sensors, Geneva, 2008.

4. R Carvell, E Usadi, N Fox, R Rusby and S Bruce, Proceedings of Sensors, Systems for Next-Generation Satellites VII, SPIE, 2004.

5. Jim Fedchak, NIST Sensor Science Division, Gaithersburg, MD, Private Communication, 7-May-2014.

6. C. D. Vaughn and G. F. Strouse, in: Proceedings of the 8th International Symposium on Temperature and Thermal Measurements in Industry and Science, TEMPMEKO 2001, B. Fellmuth, Ed., 629-634 (VDE Verlag, Berlin 2002).

7. B.W. Mangum and G.T. Furukawa, Guidelines for Realizing the International Scale of 1990. (ITS-90), NIST Technical Note 1265, 1990.

8. G. F. Strouse, Platinum Resistance Thermometer Calibrations from the Ar TP to the Ag TP, NBS Special Publication SP 250-81, U.S. Government Printing Office, Washington, D.C., (2007), 85 pp.

9. P Bramley and J Pickering, Cal Lab Magazine, 30-35, Oct 2006.

10. Isothermal Technology, Ltd., MicroK Product Brosure.

11. Sensing Devices, Inc., Lancaster, PA, Model D-100-1A, and SAMA RC21-4-1966.

12. R.P. Benedict, J.W. Murdock, ASME Trans., J. Eng. Power 235 (1963).

13. J.W. Nicholas and D.R.White, "Traceable Temperatures", $2^{\text {nd }}$ Ed., (Wiley, Chichester, 2001).

14. D R White and C L Jongenelen, Int. J. Thermophys. , 31,1685-1695 (2010).

15. ibid., The general form of the solution is applicable even under vacuum conditions for small temperature gradients, but some of the boundary conditions assume convective heat transfer, and do not apply under vacuum conditions, therefore W\&J's evaluations of the constants $K_{1}$ and $K_{2}$ (their ' $A$ ' and ' $B$ ') can not be utilized for modeling the vacuum data.

16. F.P. Incropera, D.P.DeWitt, "Fundamentals of Heat and Mass Transfer", 4th ed. (Wiley, NewYork, 1996).

17. H. Preston-Thomas, Metrologia, 27, 107 (1990).

18. L. Pitre, M. Moldover, and W. L. Tew, Metrologia, 43, 142 (2006).

19. J. Fischer, M. de Podesta, K. D. Hill, M. Moldover, L. Pitre, R. Rusby, P. Steur, O. Tamura, R. White, L.Wolber, Int. J. Thermophys. 32,12-25 (2011). 